\title{
Multi-scale Block Spectral Solution for Unsteady Flows
}

\author{
L. He \\ Department of Engineering Science, \\ University of Oxford, \\ Parks Road, Oxford, OX1 3PJ, UK
}

\begin{abstract}
Many problems of interest are characterized by two distinctive and disparate scales and a huge multiplicity of similar small scale elements. The corresponding scale-dependent solvability manifests itself in the high gradient flow around each element needing a fine mesh locally and the similar flow patterns among all elements globally. In a block spectral approach making use of the scale-dependent solvability, the global domain is decomposed into a large number of similar small blocks. The mesh-pointwise block spectra will establish the block-block variation, for which only a small set of blocks need to be solved with a fine mesh resolution. The solution can then be very efficiently obtained by coupling the local fine mesh solution and the global coarse mesh solution through a block spectral mapping. Previously the block spectral method has only been developed for steady flows. The present work extends the methodology to unsteady flows of short temporal and spatial scales (e.g. those due to self-excited unsteady vortices and turbulence disturbances). A source term based approach is adopted to facilitate a two-way coupling in terms of time-averaged flow solutions. The global coarse base-mesh solution provides an appropriate environment and boundary condition to the local fine mesh blocks, whilst the local fine mesh solution provides the source terms (propagated through the block spectral mapping) to the global coarse mesh domain. The computational method will be presented with several numerical examples and sensitivity studies. The results consistently demonstrate the validity and potential of the proposed approach.
\end{abstract}

Keywords - Multi-scale; Unsteady flow; Time-average; Unsteady source term; Block-spectral.

\section{INTRODUCTION}

Fluid mechanics problems in general are subject to a range of length scales. In many cases there is a very large and distinctive length scale disparity, which poses significant challenges to conventional computational fluid dynamics methods. Examples of practical interest include flows through porous media, effusion film cooling through many holes of a tiny size, acoustical liners in 
intakes/ducts, and surface mini-scale treatment for performance enhancement, e.g. surface microscale dimples. For the kind of problems with two distinctive scales, flow solutions are strongly influenced by the interaction between the scales. Fine scale features of a huge number of geometrically largely similar elements collectively affect globally large scale flow disturbances, while the latter set the overall environment (boundary conditions) for the former. Conventional treatment of the two-scale problems is largely empirically based, so that the micro-scale effects are modelled e.g. through modified wall boundary conditions, whilst the large scale disturbances are solved. It is recognized that the behavior of the problems of interest may well be considerably dependent on detailed geometrical features of micro-scale element configurations. We would thus like to be able to understand and predict such influences arisen from the small scale geometry changes. In particular, the development of predictive capabilities for the two-scale problems should have a potential appeal for wide future applications given the rapid development in the Additive Manufacturing (AM) technologies.

Various multi-scale methods and techniques have been developed and applied for problems of distinctive scale disparities spatially for various flow regimes (e.g. [1], [2], [3], [4]) or temporally for fluid-solid transient thermal coupling (e.g. [5], [6], [7]). More specifically a block spectral modeling technique has been recently developed to address the two conflicting requirements in the problems with two distinctive spatial length scales: a) high resolution to capture relevant small scale flow features and b) avoidance of having to have very fine resolution globally for a large domain. The validity and effectiveness of the block spectral approach have been examined in test cases for films cooling ([8]), dimples and lined acoustic intakes ([9][10]). A main restriction of the previous block spectral formulation is that the flow to be solved is assumed to be steady, or quasi-steady in that unsteadiness is allowable only if it is of a large scale. It is recognized that the previous block spectral method is unable to deal with unsteadiness either when the scale of unsteadiness is comparable to that of the small sub-domains, or when it arises from self-excited unsteadiness (e.g. vortex shedding and turbulence eddies). This is because in these unsteady cases, the smoothness of the block-to-block variation as assumed for the block spectrum would be lost, thus the local unsteady solutions in those solved local small blocks are not readily propagatable to the global large domain. This inability in dealing with small scale self-excited unsteadiness presents a major limitation of the previous formulation of the block spectral method, 
particularly given increasingly progressive developments and applications of turbulence eddy resolved methods, Large Eddy Simulations (LES)/ Direct Numerical Simulation (DNS).

The primary consideration of the present work in extending the block-spectral method to unsteady flows stems from linking an unsteady flow to its time-averaged counterpart. For example, consider a flow passing through a multiple row configuration with each row having 3 cylinders. The instantaneous unsteady flow field (Fig.1a) is clearly subject to short scale and seemingly irregular disturbances which make it very difficult to map a local flow solution around one cylinder to those around others, even though all the cylinders are of the same geometry and relative spacing. On the other hand, the time-averaged flow (Fig.1b) is much smoother (thus effectively of much longer length scales in terms of the cylinder-to-cylinder variation) than its instantaneous unsteady counterpart. The contrasting characteristics suggest that the time-averaged flow should be far more amenable to the block spectral treatment.

The potential in working with a time-averaged flow field has been extensively and simply illustrated in the common Reynolds averaging procedure for turbulent flows. The time-averaged turbulent flow can be solved by a steady flow solver if the extra product terms due to the nonlinearity of advection terms (i.e. 'Reynolds stresses') are given (modelled or solved). The procedure should be applicable to general (random or periodic) unsteady flows as long as a timeaveraged state exists. For externally driven periodic unsteady flows due to relative rotor-stator bladerow motions in turbomachinery, the interaction between the unsteady and time-average ('steady') parts is included in terms of 'deterministic stresses' after Adamczyk [11]. More closely relevant to the present interest in dealing with self-excited short scale unsteadiness is the work of Ning and He [12], showing that by adding 'unsteady stresses' as extra momentum and energy source terms, it would be possible to obtain the time-averaged flow field with a purely steady flow solver for self-excited vortex shedding around a cylinder or a blade trailing edge. This 'unsteady stress' method has been so far however only applied to self-excited unsteady flows for simple and isolated geometrical configurations (Ning and He, [12], Lokovic et al [13]).

The primary objective of the present work is to develop an efficient and accurate method for getting time-averaged flow solutions of self-excited unsteady flows in configurations with a large number of similar micro-structures. This will be built on the two existing elements of computational capability as reviewed above: the block spectral approach as demonstrated for 
steady flows [8], [9] [10] and the potential of getting a 'steady' solution for a time-averaged flow by means of 'unsteady stress' terms [12]. In the following sections, the basic flow decomposition and two-scale modelling formulations will be first introduced. The feasibility of getting a timeaveraged flow solution by adding the unsteady source terms is demonstrated, with a measure introduced to enhance the convergence to a steady state by an additional numerical damping. A further refined formulation for propagating the source terms from the local discrete fine mesh block domains to a global coarse mesh domain will then introduced, followed by several validation and sensitivity case studies.

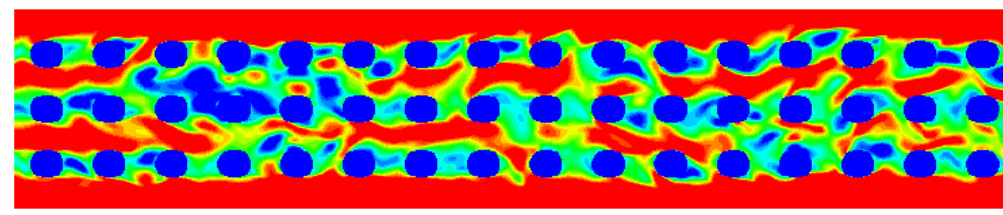

(a) Unsteady

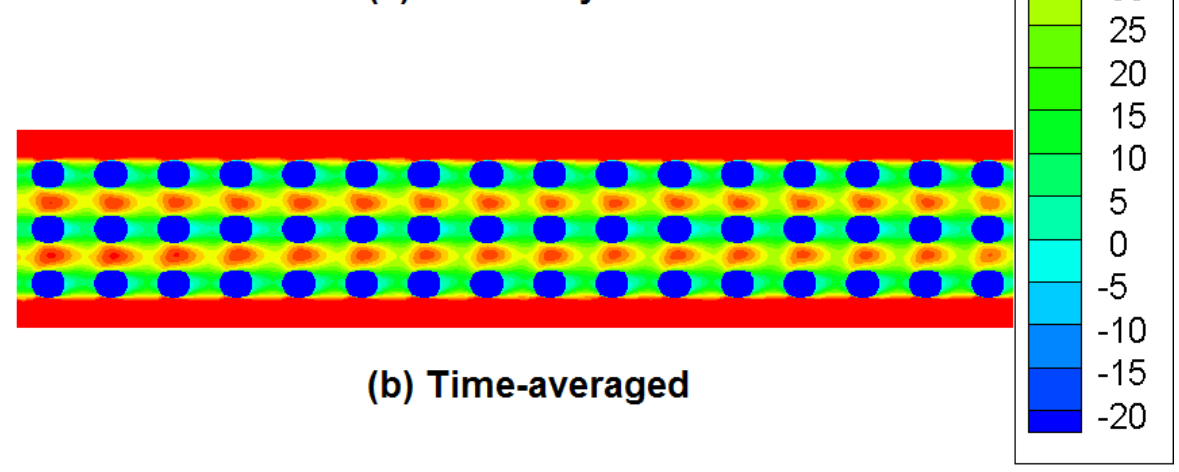

Fig.1 Unsteady Flow vs Time-averaged Counterpart (in m/s) (Contours of velocity in the streamwise direction for flow around multiple cylinders)

\section{BASELINE FLOW SOLUTION METHOD}

Consider a flow governed by the 3 dimensional compressible unsteady Navier-Stokes equations, written in a simple form:

$$
\frac{\partial W}{\partial t}+R(W)=0
$$

where $\mathrm{W}$ is the 5 element vector of conservative flow variables. $\mathrm{R}$ is the lumped advection and diffusion terms. 
The computational domain is discretized with a multi-block structured mesh. The equations are spatially discretized using a cell center based finite volume scheme. The spatially discretized equations are numerically integrated in time using an explicit 4-stage Runge-Kutta scheme which can be used for both steady and unsteady flows. For an unsteady solution with fine meshes where time step sizes limited by the numerical stabilities are too restrictive, the equations are solved by a $2^{\text {nd }}$ order implicit dual timing scheme after Jameson [14] . Introducing a pseudo time $\tau$, a semi discrete form of the flow equations for an unsteady solution at physical time step $\mathrm{N}$ should be:

$$
\left(\frac{\partial W}{\partial \tau}\right)^{k}+\frac{1.5 W^{N, k}-2 W^{N-1}+0.5 W^{N-2}}{\Delta t}+R\left(W^{N, k}\right)=0
$$

The $2^{\text {nd }}$ order accuracy of the temporal approximation is fully recovered when the pseudo time marching iterations (indexed by pseudo time step k) converge at the given physical time step. Alternatively the unsteady solution can be obtained by a direct explicit time marching. Given the time step size being limited by the minimum mesh spacing as required by the numerical stability, the physical time step can be augmented to a certain extent for cases with non-uniform mesh spacing by using the time consistent multi-grid [15].

For the spatial discretization of the equations, a $2^{\text {nd }}$ order central difference type of spatial discretization was previously adopted in the solver for evaluating the fluxes at the cell boundary faces [15] [16]. Recently, a pressure-advective flux split upwinding discretization, AUSM (Liou [17]) has been implemented. The upwind scheme is shown to produce less numerically dissipative results in near-wall viscous flow regions, as desired. The baseline implementation of the AUSM is 2nd order accurate in space for a uniformly regular mesh, following van Leer's MUSCL formulation. The $2^{\text {nd }}$ order flux calculations need flow variables to be stored at 5 mesh cell center points.

A relatively simple extension to a $3^{\text {rd }}$ order spatial accuracy has been adopted, following Cada and Torrilhon [18]. It has been shown that a $3^{\text {rd }}$ order accurate flux calculation can be realized in a smooth flow regime by a suitable formulation of the nonlinear limiter. The main appeal of this formulation is that a compact stencil formulation in the limiter calculation is equivalent to only needing the variables from 5 mesh cell centers for flux calculations on a mesh cell face. It is the same number of mesh points involved as the $2^{\text {nd }}$ order scheme, though the limiter calculations are slightly more time-consuming. For the present multi-scale method, a use of different levels/fidelities of discretization for different zones is particularly desirable. 
The inlet/exit far field boundary conditions can be given by the compatibility conditions: specification of the inlet stagnation pressure and temperature, the inflow angle, and the exit static

pressure. Alternatively, the local characteristics based non-reflective boundary conditions are used. On a solid surface, a nonslip wall condition is used for a viscous wall, or a symmetry condition for an inviscid wall boundary.

It should be noted that the baseline solver has the one-equation model of Spalart-Allmaras [19] and the two-equation k- $\omega$ SST model of Menter [20] as options for turbulence closure when a RANS mode is run. Most solutions for the present test cases will however be run in a laminar mode and unsteadiness will be purely self-excited.

\section{SOLUTION OF TIME-AVERAGED FLOW}

\subsection{Flow Decomposition for Time-Averaged Flow Equations}

The present method framework starts with a split of unsteady flow into a time-averaged part and a fluctuating one:

$$
W(x, t)=\quad \bar{W}(x)+W^{\prime}(x, t)
$$

Time-averaging the original flow equations (Eq.1) gives,

$$
\overline{R(W)}=0
$$

Due to nonlinearity of the advective fluxes, one recognizes, $\overline{R(W)} \neq R(\bar{W})$.

We denote the difference between the time-averaged lumped fluxes and the lumped fluxes calculated using the time-averaged flow variables as 'unsteady stress terms' (UST), as by Ning and He [12]. In particular, note that these extra terms can contain contributions from all kinds of self-excited unsteady disturbances including periodic vortex shedding and random turbulence eddies. We also include the nonlinearity resultant terms from the dissipation terms (e.g. due to nonlinear relations of viscosity and conductivity coefficients with temperatures), though this nonlinear effect tends to be much smaller than that of the advection terms. As a result, the time averaged equation is written as,

$$
\overline{R(W)}=R(\bar{W})+U S T=0
$$


The equation above (Eq.5) effectively provides a very convenient way of calculating the UST terms, as we only need to calculate the flux residuals using the time-averaged flow variables which can be easily obtained with a simple moving-average shown to be effective for a flow subject to un-deterministic flow instabilities with unknown frequencies in prior by He [21]. For physical time step $\mathrm{N}$, a time-averaged variable can be updated by the old value at $\mathrm{N}-1$ and that from the current solution:

$$
\bar{W}^{N}=\frac{N-1}{N} \bar{W}^{N-1}+\frac{1}{N} W^{N}
$$

Thus, the UST at time step $\mathrm{N}$ can be simply obtained by calculating the advection and diffusion terms using the time-averaged flow variables obtained up to this time instant:

$$
U S T^{N}=-R\left(\bar{W}^{N}\right)
$$

\subsection{Convergence Enhancement with Balanced Artificial Eddy Viscosity Damping}

All the nonlinear product terms for the momentum and energy equations, mimicking the Reynolds stress and flux terms in RANS arisen from turbulence fluctuations, can be simply lumped together, denoted as 'unsteady source terms' (also 'UST'). The source terms for the continuity equation should be negligibly small when the conservative flow variables are used for time-averaging (i.e. the Favre-averaging).

Time marching in the pseudo time $\tau$ to solve the time-averaged flow equation, we have.

$$
\frac{\partial W}{\partial \tau}+R(W)=-U S T
$$

When converged to a steady state, this should lead to the solution of the time-averaged flow field satisfying the time-averaged flow equation (Eq.5).

As shown by Ning and He [12], the UST provides some considerable damping to suppress the unsteadiness so that a steady solution becomes possible. Given the physical origin of the unsteadiness, the effectiveness of the damping should depend on numerical schemes and meshes adopted. For instance, for a more dissipative numerical discretization scheme or an under-resolved mesh for small scale self-excited unsteadiness, the UST may provide sufficient dissipation and damping for getting a fully converged steady solution. The numerical tests in the present work have shown that the damping effect of the UST alone often tends to be insufficient for reaching a 
good convergence to a steady solution. A similar observation has been made by Zhang [22], where an extra damping in the form of the one-equation Spalart-Allmaras turbulence model [19] was introduced.

Here a simple mixing-length based artificial eddy viscosity (AEV) in a similar form to that by Denton [24] is introduced to deal with this issue:

$$
\begin{array}{ll}
\text { し }=\text { ky } & \left(\mathrm{y}<\mathrm{L}_{\mathrm{d}}\right) \\
\text { し }=\mathrm{L}_{\mathrm{d}} & \left(\mathrm{y}>\mathrm{L}_{\mathrm{d}}\right)
\end{array}
$$

where $\mathrm{k}$ is the von Karman constant, $0.41 ; \mathrm{y}$ is the distance to the wall and $\mathrm{L}_{\mathrm{d}}$ is a cut-off mixing length to be empirically specified. The corresponding artificial eddy viscosity for the extra damping is,

$$
v_{D}=\iota^{2}|\bar{\omega}|
$$

Given the artificial nature of this damping in general and the empirical input of the cut-off mixing length in particular, the extra damping term will have be rebalanced to counter the negative impact on solution accuracy. The artificial eddy viscous dissipation is in the same form as that of the corresponding physical viscous diffusion terms. The key constraint is that when converged, the original time-averaged flow equation should be recovered. Thus the equations are modified with the added damping terms ' $\mathrm{D}$ ' in a form of,

$$
\frac{\partial W}{\partial \tau}+R(W)+U S T=D(W)-D(\bar{W})
$$

The first term of RHS of the equation above provides the extra damping instantaneously in a form of the standard viscous dissipation form but with the extra AEV $v_{D}$ (Eq10) to the current solution. The last term of RHS is obtained from the time-averaged solution over the time period up to the present time instant. When the solution converges to a steady state, the time-averaged flow field will be produced. $D(W)$ then approaches $D(\bar{W})$, thus the two damping terms will be balanced out and the time-averaged equation (Eq.5) will be completely recovered. The maximum cutoff mixing length is an empirically input parameter. However, the way the balanced damping is introduced should be able to minimize the uncertainty, as will be illustrated in the example next. 


\subsection{Results for Flow around Cylinder}

A simple but fundamental case for testing the unsteady flow modelling capability is the laminar flow around a cylinder. Most of classic results are for a low speed incompressible flow. The present compressible flow solver is run at a low speed inflow condition taken to be $55 \mathrm{~m} / \mathrm{s}$. to ensure the maximum velocity around the cylinder is still within an incompressible flow regime.

The Reynolds number based on the cylinder diameter is taken to be 174 , to match one of the experimental cases in Thom's pioneering classic study [24]. This is about the upper limit of Re when flow is both fully laminar and periodically unsteady due to vortex shedding. The instantaneous entropy contours from the direct unsteady solution are shown in Fig. 2. The reduced frequency (Strouhal number) from the present calculated unsteady flow is 0.196 , in good agreement with the value of 0.2 from well-established correlations for this Reynolds number. The time-averaged surface pressures are plotted in terms of the pressure coefficient variation with the circumferential angle of the polar coordinate system (Fig.3). The calculated results are in agreement with Thom's experimental data [24].

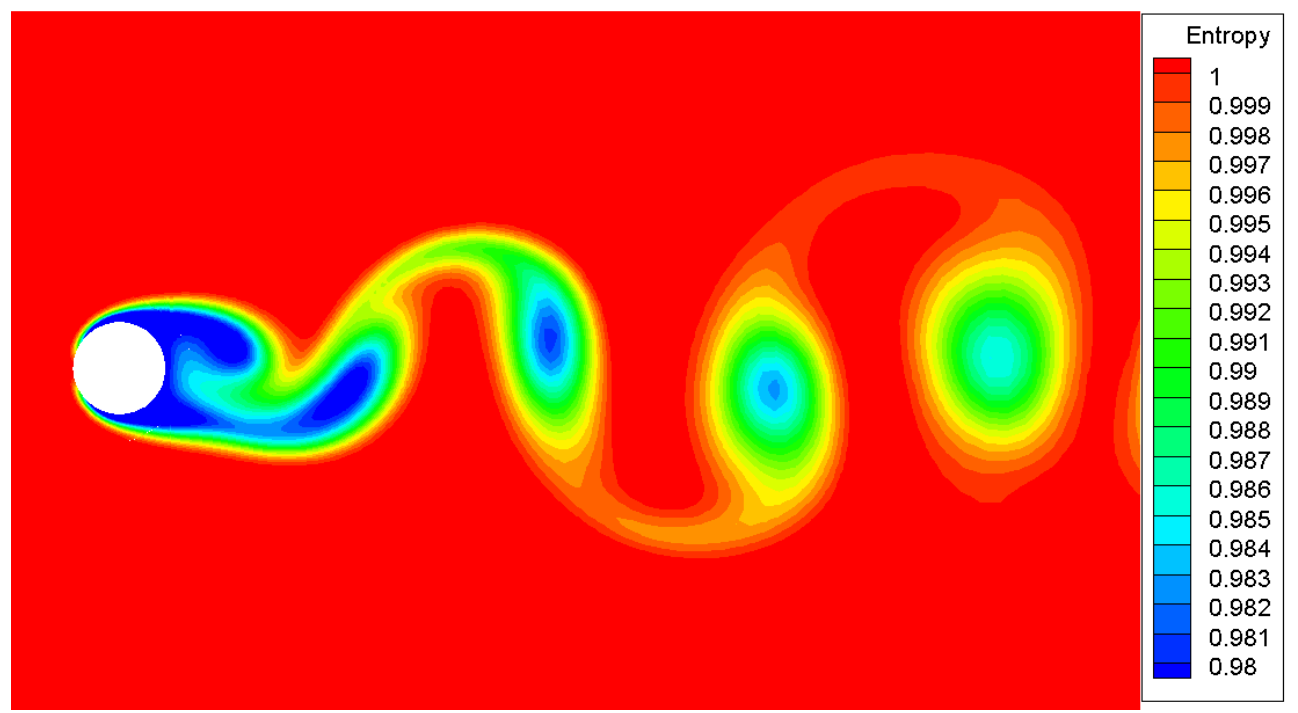

Fig.2 Instantaneous Entropy Function $[\exp (-\Delta S / R)]$ for Flow around a Cylinder 


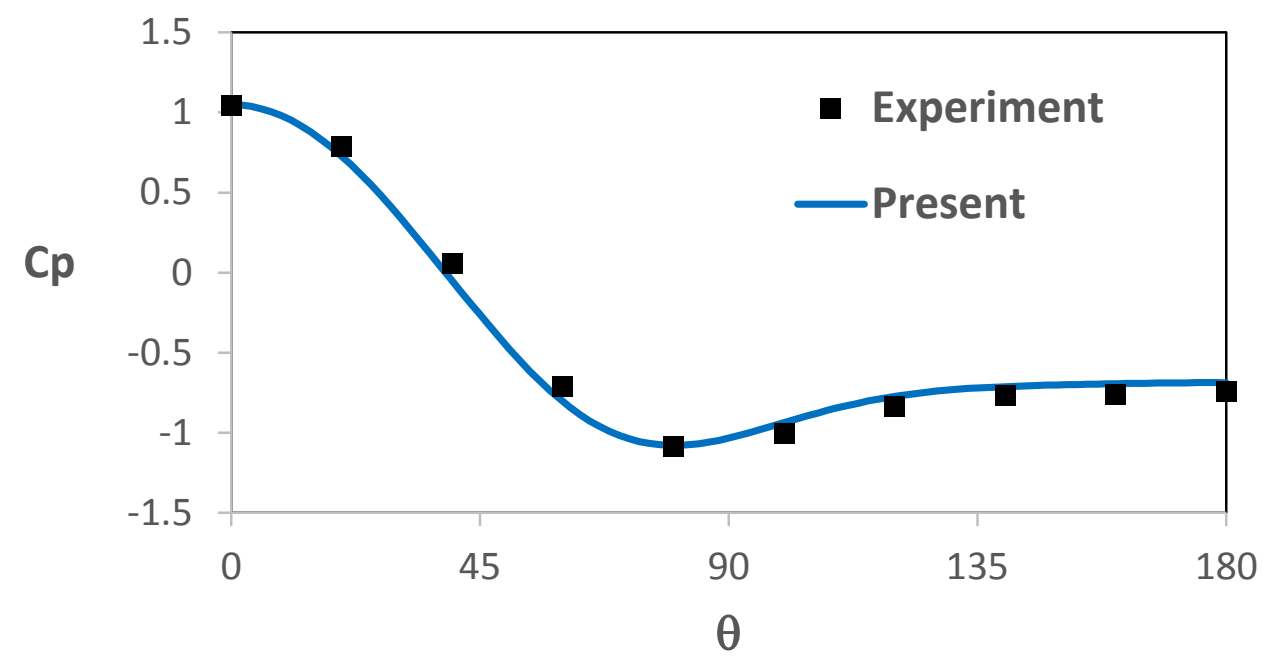

Fig.3 Time averaged Pressure distribution along cylinder surface ( $\theta=0$ - leading edge; $\theta=180^{\circ}$ - trailing edge) compared to Thom's experiment [24]

The impact of the unsteady source terms on the time-averaged solution convergency and accuracy is now examined. The lumped unsteady stress terms are obtained from the direct unsteady solution, and are added to the flow equations for the corresponding mesh cells as source terms. Fig. 4 shows the time histories of the maximum $\mathrm{x}$ velocity change between two consecutive time steps. These are equivalent convergence histories when a steady flow solution is pursued. Four cases are compared:

1) The solution without the UST source terms;

2) The solution with UST terms but zero artificial viscosity damping term $\left(\mathrm{L}_{d}=0\right)$

3) The solution with UST terms and an artificial viscosity damping term $\left(\mathrm{Ld}_{\mathrm{d}}=0.1 \mathrm{D}\right)$

4) The solution with UST terms and a larger artificial viscosity damping term $\left(\mathrm{L}_{\mathrm{d}}=0.2 \mathrm{D}\right)$

where $\mathrm{D}$ is the cylinder diameter. It can be seen that the addition of the unsteady stress terms does provide some stabilizing effect but the residuals can only be dropped by about one order of magnitude for the present numerical scheme and mesh adopted. It is also clear that as expected, the convergence can be significantly enhanced by the inclusion of the artificial eddy viscosity (AEV) damping term. 


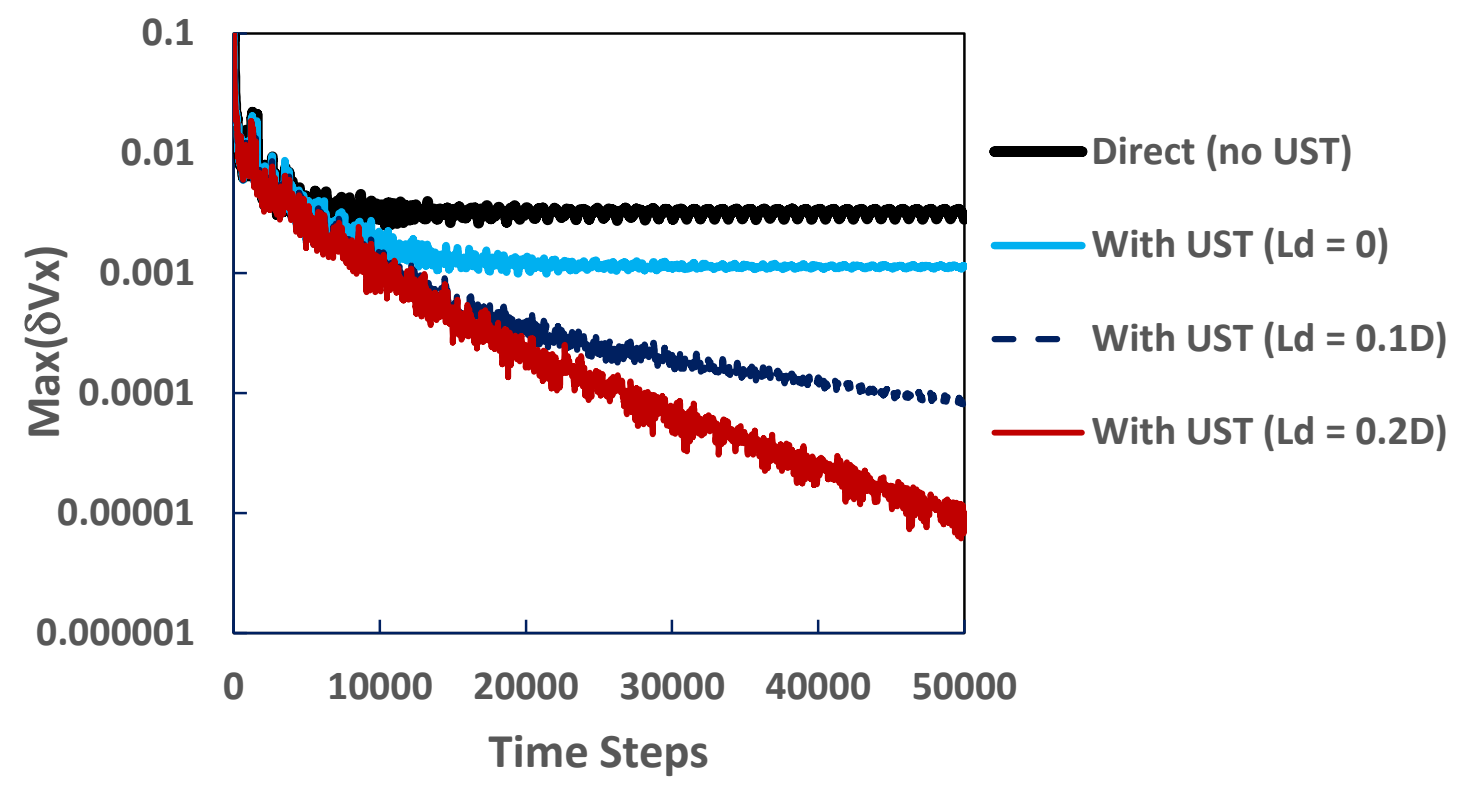

Fig.4 Convergence Histories for Different AEV Cut-off Mixing Lengths

The main issue of interest is the influence of the AEV damping on the solution accuracy. Fig.5 shows the comparison of time averaged $\mathrm{x}$ velocity contours among the solutions with different cutoff mixing lengths. Also included in Fig.5 is the baseline direct solution as a reference. Firstly, we note the good agreement in the time-averaged flow field between the direct unsteady solution and the present steady ('quasi-steady') solutions with the unsteady source terms. This again illustrates the validity of the basic source term approach for self-excited flows as first indicated by Ning and He [12]. They also illustrated markedly different results from the equivalent steady solution without the source terms.

We can also see from Fig.5 that the AEV damping, because of the way of the balanced formulation (Eq.11), shows hardly any influence on the solution accuracy. When the solution is fully converged, the time-averaged solution becomes time-independent as the cases with the AEV. The insensitivity of the results to the empirical choice of the cutoff mixing length as intended is also indicated by the comparison of the time-averaged surface pressures (Fig.6).

The results of this case study for the flow around a single cylinder illustrate the validity of the unsteady source term (UST) procedure, and form a sound basis for the present work in extending the block spectral method to unsteady flow solutions. 


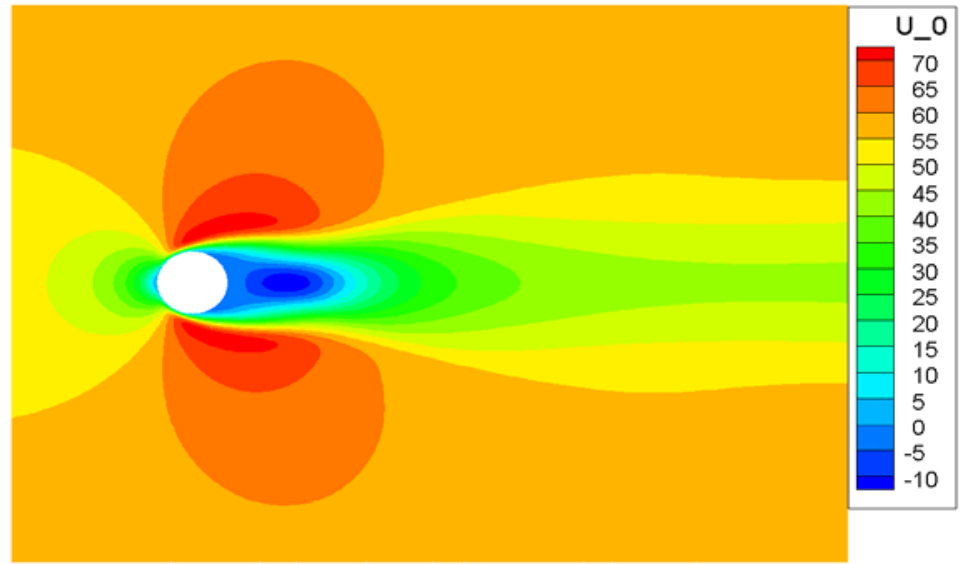

(a) Direct Unsteady Solution

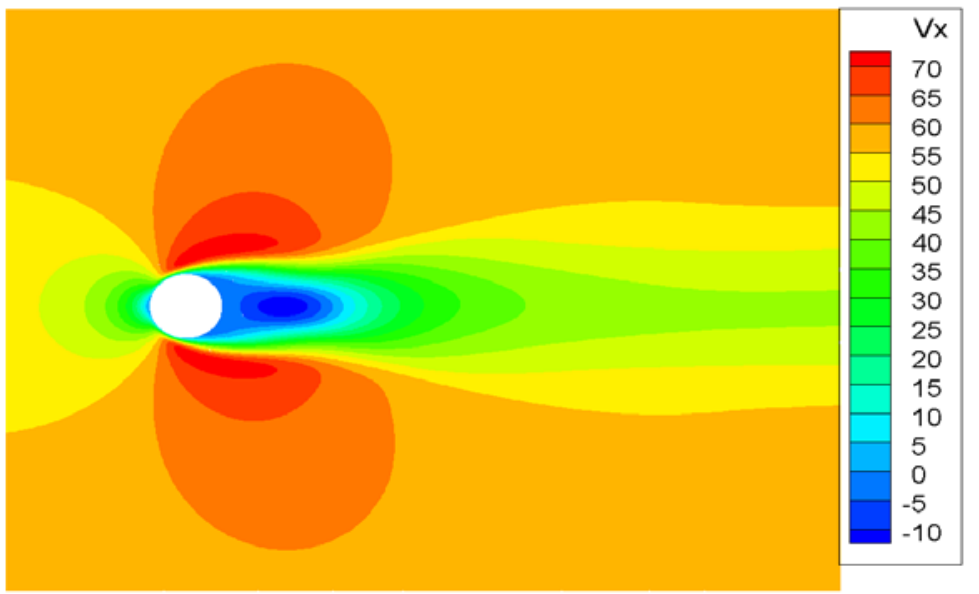

(b) Steady + Source (AEV damping: $\mathrm{Ld}=0.1 \mathrm{D})$

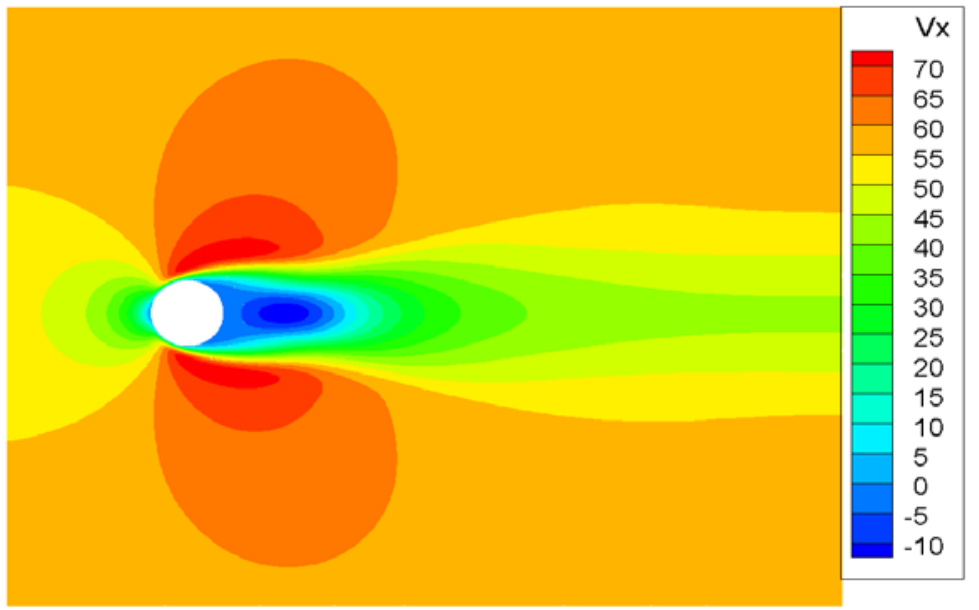

(c) Steady + Source (AEV Damping: Ld=0.2D)

Fig.5 Comparisons of Time-averaged Axial Velocities with AEV Damping 


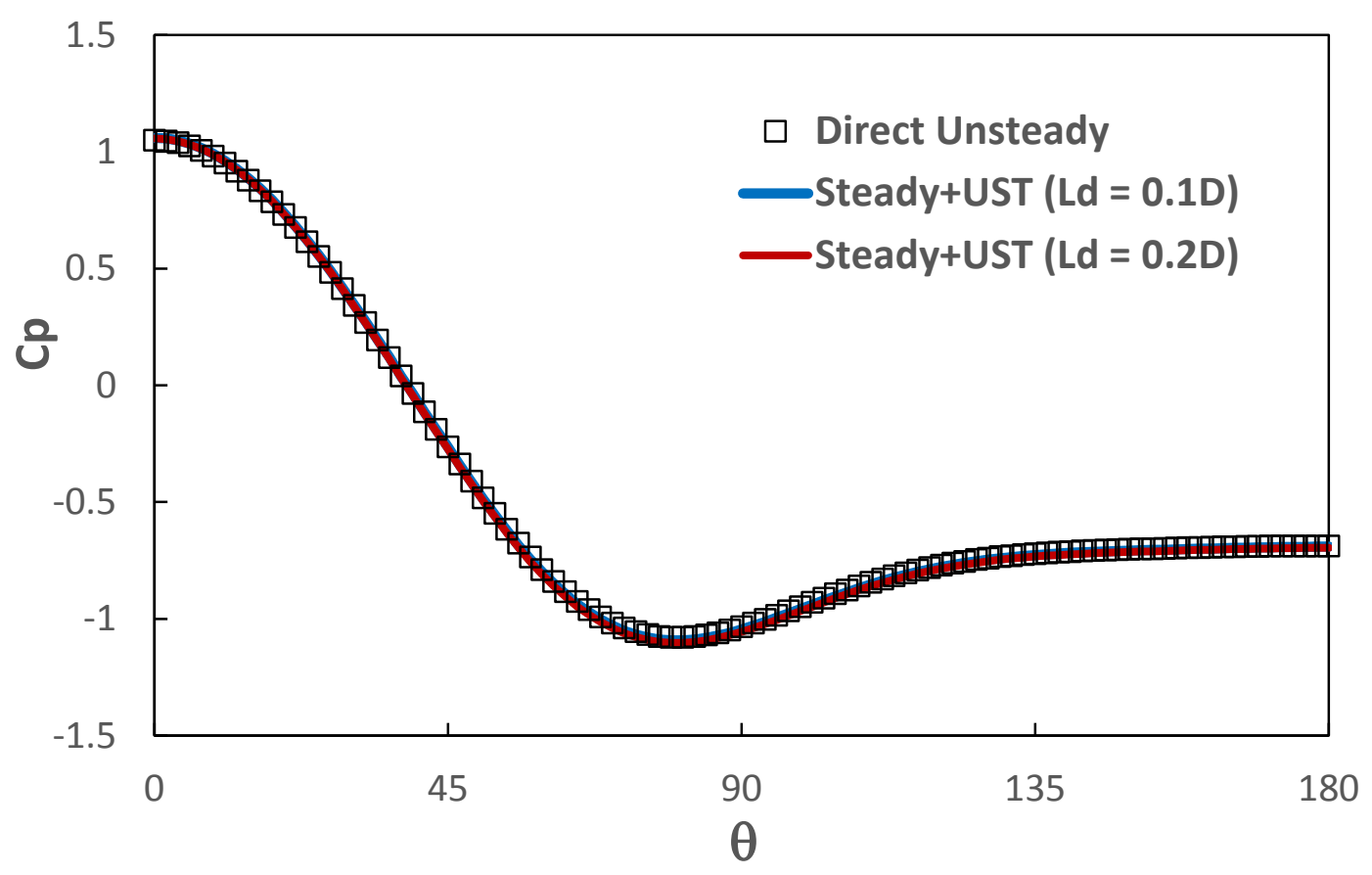

Fig.6 Comparisons of Time-averaged Surface Pressure Distributions

\section{BLOCK SPECTRAL SOLUTION FOR UNSTEADY FLOW}

The basis for the block spectral methodology is the scale-dependent solvability. The flow field of a global domain consists of a large number of small block domains of similar geometrical and flow patterns. Thus although each block is subject to complex local flow features, there should be a relatively smooth block-to-block variation, to be effectively and accurately approximated by a spectral representation. The block spectral method takes the advantage that only a small number of discrete fine mesh blocks need to be solved to construct the spectrum, particularly appealing for solving problems with a large number of micro-structure elements of high flow gradients locally but a similar flow pattern among the structure elements. The validity and effectiveness has been checked and demonstrated for steady flows [8] [9] [10]. Given the feasibility of achieving a time-averaged flow solution through the unsteady source terms for a given mesh as shown in the previous sections, we now consider to make use of the block spectral method to get a time-averaged flow solution of a large coarse mesh domain by means of high resolution unsteady solutions from a small set of fine mesh blocks. To this end, there are three related aspects to be dealt with, as will be described in the following sections: 
1) Suitably embedded fine mesh block domains and treatment of the interfaces of these blocks with the base mesh domain.

2) Suitable forms of the coarse mesh flow equations with appropriate source terms.

3) Mapping of the source terms from the local fine mesh blocks to the global coarse mesh domain.

\subsection{Discretely Embedded Dual-mesh Domain}

The selection of a multi-scale computational mesh needs to fulfill two basic requirements:

i) to capture global large scale flow disturbances to provide effectively the right environment (boundary conditions) for the local fine-mesh domain solutions, and

ii) to resolve local high gradient small scale disturbances.

For i), only a coarse mesh to cover the whole domain would be needed. But a direct solution on this coarse mesh alone will miss the influence of flow disturbances to be captured only by the fine mesh. For ii), a fine mesh would be needed, but the scale-dependent solvability means that we will only need a local fine mesh for resolving the high gradient within a micro-structure element. The local fine mesh solution however can only be meaningful if it is subject to a right environment (boundary conditions). Therefore the global coarse mesh solution and the local fine mesh solution are inherently linked.

Given the above consideration, a dual-mesh is taken in the present work. Consider a computational domain for flow around a large number of regular micro structures. A coarse base mesh is used to discretize the whole domain. A fine mesh block will be simply embedded based on a micro-structure. For example, consider a dual mesh for a domain with a large number of cylinders. Six local fine mesh blocks are taken to be discretely embedded in a base-mesh domain, each containing 4 cylinders, as shown in Fig.7. 


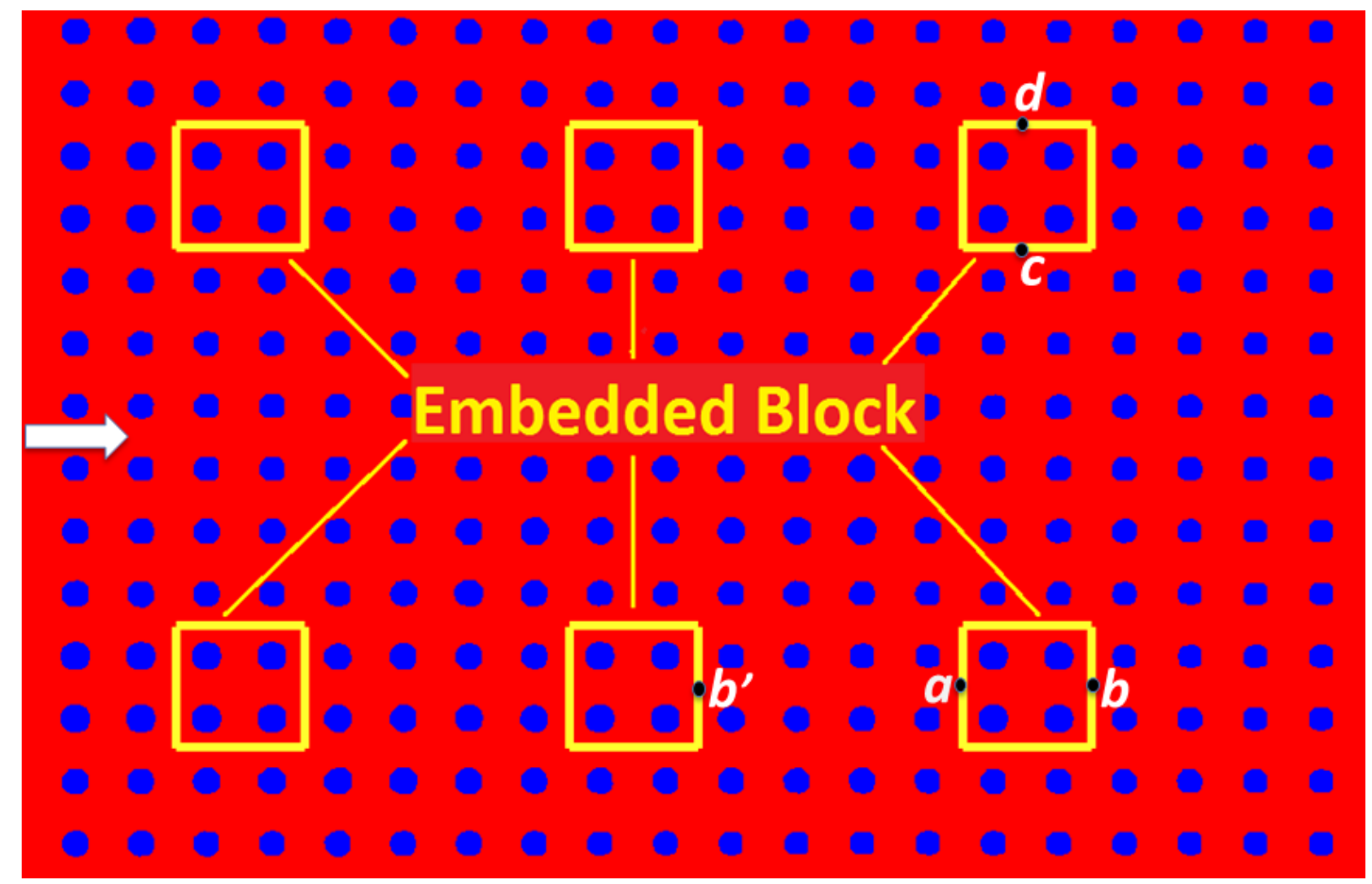

Fig.7 Base Mesh Domain with 6 Discretely Embedded Mesh Blocks (inflow from left)

The embedded local domains are subject to a fine mesh solution. The flow variables of the corresponding embedded base coarse mesh cells are locally overwritten by (directly transferred from) those from the fine mesh blocks. This is similar to the immersed mesh block method for film cooling analysis (Lad and He [25]). The transfer of the fine mesh solution $\mathrm{W}_{\mathrm{f}}$ to that of the coarse mesh $\mathrm{W}_{\mathrm{C}}$ is based on a simple volume-weighted average:

$$
W_{C}=-\frac{1}{\Delta V_{c}} \sum W_{f} \Delta V_{f}
$$

where the summation is taken for all the fine mesh cells (of volume $\Delta V_{f}$ respectively) embedded in a coarse mesh cell of volume $\Delta V_{C}$.

Considerations are now given to treating the interfaces between the base mesh side and the fine mesh boundary. Firstly the flux conservation requirement manifests as that the coarse mesh fluxes over boundary mesh cell area $\Delta A_{c}$ are overwritten by their area-weighted counterparts from the overlapping fine mesh side:

$$
\operatorname{Flux}_{C}=-\frac{1}{\Delta A_{c}} \sum \operatorname{Flux}_{f} \Delta A_{f}
$$


Secondly, the updating of the dummy mesh points of an embedded block boundary needs to take into account the different mesh resolution between the two sides. A flow variable at a dummy point of the fine mesh block boundary is split into two parts, a base mesh resolved component and a fluctuation corresponding to the fine mesh resolution:

$$
W(x, t)=W_{C}(x, t)+w_{f}^{\prime}(x, t)
$$

$W_{C}$ is the base coarse mesh resolved part, directly interpolated from the surrounding coarse mesh points. The small scale fluctuating disturbance $w_{f}^{\prime}$, subject to the fine mesh resolution, can then be taken from a corresponding mesh point from its counterpart boundary. The treatment of the fine mesh fluctuation part is similar to an inflow turbulence generation at an upstream boundary by recycling the unsteadiness from a downstream boundary counterpart. For instance, given an inflow from the left to the base mesh domain as shown in Fig7, $\boldsymbol{a}$ is an upstream point with its downstream counterpart point $\boldsymbol{b}$ for the same fine mesh block. The corresponding implementation of the recycling condition will be:

$$
\left[w_{f}^{\prime}(x, t)\right]_{a}=\left[w_{f}^{\prime}(x, t)\right]_{b}
$$

An alternative option is to update the fluctuation of point $\boldsymbol{a}$ with its counterpart from an upstream domain, $\boldsymbol{b}$ '. This would help de-correlate the points within the same fine mesh block. A similar interface condition can be applied for the fluctuations between two streamwise parallel ('periodic') boundaries, e.g. between points $\boldsymbol{c}$ and $\boldsymbol{d}$ (Fig.7).

It must be emphasized that this recycling type interface treatment is only applied to the fine mesh resolved fluctuation part $w_{f}^{\prime}$, as the base mesh resolved variable $W_{C}(x, t)$ at one boundary will be unlikely to be the same as that at the counterpart boundary. The base mesh flow variable $W_{C}(x, t)$ at an upstream boundary point of a block domain will certainly not be the same as that at its downstream counterpart.

\subsection{Base-mesh Domain Solution with Source Terms}

Given the dual mesh domain with embedded fine mesh blocks, some further considerations need to be given to the source terms in relation to the coupling between the base mesh and embedded mesh solutions. Firstly, we note the difference in spatial resolution between the two mesh regions, in addition to the difference due to temporal resolution as discussed earlier between a direct unsteady equation and its time-averaged counterpart for the same mesh. Furthermore, we 
need to consider the convergence behavior when a steady solution for the base mesh domain is pursued, as discussed in 3.2 regarding the additional numerical damping in the form of the artificial eddy viscosity (AEV). Although the additional damping would enhance the convergence to a steady state, it is unclear how the artificial damping term may be propagated from the local fine mesh solution to the global base mesh solution without causing further errors. It is therefore preferred to pursue the multi-scale solution on the dual mesh without evoking the artificial numerical damping. This means that the base mesh solution even with the source term may still contain certain unsteadiness, not fully converging to a steady state. As such, the residual unsteadiness in the base coarse mesh solution will have to be taken into account when formulating the source terms to be added to the flow equation.

Consider first the flow solution of an embedded fine mesh flow field in relation to the corresponding base-mesh flow field. The difference between the fine mesh and the coarse mesh solutions is a manifestation of the influence of the fine mesh solution. The fine-to-coarse mesh transfer (Eq.12) at any time instant during an unsteady solution process can be regarded as a spatial averaging, thus the coarse mesh flow variables will not satisfy the flow equations if directly discretized on the coarse mesh. Similar to the unsteady stress terms, the instantaneous unsteady equation with the coarse mesh flow variables can be balanced only when extra source terms due to the spatial averaging (denoted as Spatial Resolution Source Term, SRST') are introduced, i.e.

$$
\frac{\partial W_{C}}{\partial t}+R_{C}\left(W_{C}\right)+S R S T^{\prime}=0
$$

Given that the time-averaged flow is to be targeted, time-averaging Eq.16 gives:

$$
R_{C}\left(\overline{W_{C}}\right)+U S T+S R S T=0
$$

where SRST is the time-averaged SRST', and UST is the unsteady source terms due to the timeaveraging as before. Both the temporal and spatial contributions to the source term can be lumped together, denoted simply as Total Source Terms from the fines mesh solution ('TST ${ }_{\mathrm{f}}$ '). The timeaveraged equation for the coarse mesh domain then simply becomes.

$$
R_{C}\left(\overline{W_{C}}\right)+T S T_{f}=0
$$

It must be remarked that Eq.18 serves different functions in different parts of the coarse mesh domain. For an embedded mesh block region where the coarse mesh solution is directly transferred 
from the fine mesh solution (Eq.12), the equation will simply be used to obtain the source term $\mathrm{TST}_{\mathrm{f}}$. Thus for this part of the coarse mesh region, Eq.18 is rewritten as.

$$
T S T_{f}=-R_{C}\left(\bar{W}_{f-C}\right)
$$

where subscript ' $\mathrm{f}-\mathrm{C}$ ' denotes the flow variables on the coarse mesh which are directly transferred from the corresponding embedded fine mesh solution using Eq.12.

For other non-embedded base mesh regions, the local coarse mesh solution will need to be balanced with the added source terms to be propagated by the block spectral mapping as described in the next section. Eq.18 will then be the target equation to govern the corresponding timeaveraged flow of the local coarse mesh solution.

Given these considerations, we can now determine the total source terms to be added to the unsteady flow equation for a non-embedded coarse mesh region $\left(\mathrm{TST}_{\mathrm{C}}\right)$ :

$$
\frac{\partial W_{C}}{\partial t}+R_{C}\left(W_{C}\right)+T S T_{C}=0
$$

Time averaging Eq.20 gives:

$$
R_{C}\left(\overline{W_{C}}\right)+U S T_{C}+T S T_{C}=0
$$

Comparing the equation above (Eq.20) to the target coarse mesh equation (Eq.18), we then have,

$$
T S T_{C}=T S T_{f}-U S T_{C}
$$

The coarse mesh unsteady stress term (USTC) is the difference between the time-averaged fluxes and the fluxes simply calculated using the time-averaged variables of the local coarse mesh solution:

$$
U S T_{C}=\overline{R_{C}\left(W_{C}\right)}-R_{C}\left(\overline{W_{C}}\right)
$$

Effectively, the time-averaged solution of the coarse mesh domain will be subject to an unsteady flow equation with the added total source terms:

$$
\frac{\partial W_{C}}{\partial t}+R_{C}\left(W_{C}\right)=-T S T_{f}+U S T_{C}
$$

Note that the RHS of the above equation will all only depend on the time-averaged flow solutions, the first term comes from the time-averaged fine mesh solution (Eq.19), and the second from the time-averaged coarse mesh solution (Eq.23). Most importantly, we note that time-averaging Eq.24 
will lead to the exact form of the target steady equation (Eq.18) governing the time-averaged flow for the coarse mesh domain, as intended.

Thus, as long as we can provide the coarse mesh domain with a source term from the fine mesh domains ( $\left.T S T_{f}\right)$, the time-averaged solution on the coarse mesh domain should then be equivalent to that directly transferred from a local fine mesh solution. Passing the source terms from the fine mesh solution to the coarse base-mesh one in the form of the augmented unsteady flow equation (Eq.24), we now can completely avoid adding any artificial damping terms as described in section 3.2. The target steady flow equation (Eq.18) governing the time-averaged flow on the coarse base-mesh will be recovered regardless of the corresponding coarse mesh solution being steady or unsteady. As a fully converged steady solution is no longer needed, there is thus no need for the AEV damping. The only requirement now is the existence of a time-averaged flow state for the base-mesh domain.

\subsection{Block Spectral Mapping of Source Terms}

As introduced, the previous block spectral method has been examined and demonstrated for steady and long wavelength quasi-steady flows. Recognizing that the time-averaged field of an unsteady flow is much smoother than its instantaneous counterpart (Fig.1), we now extend the block spectral methodology to mapping time-averaged variables from local embedded fine mesh domains to a global coarse mesh domain. A mesh-pointwise smooth variation among the fine mesh blocks is subject to a block-block spectral approximation.

The fine mesh blocks with similarity are often distributed in a two dimensional manner, e.g. for geometrically similar micro-structures on a solid surface. Then a huge number small and similar mesh blocks need to be taken if solved directly. Here we would instead like to establish the block-block spectral variation of a flow variable by solving only a much smaller set of fine mesh blocks. Then with the block-block spectrum, we will be able to get the distribution of the flow variables for the global mesh domain.

By now we have established that the variables to be obtained from the embedded fine mesh

blocks and mapped to the global coarse mesh domain would be the total source terms (TST) based on the time-averaged solution from the discretely embedded fine mesh blocks (Eq.19). We consider 2-dimensionally distributed blocks as shown in Fig.8. 


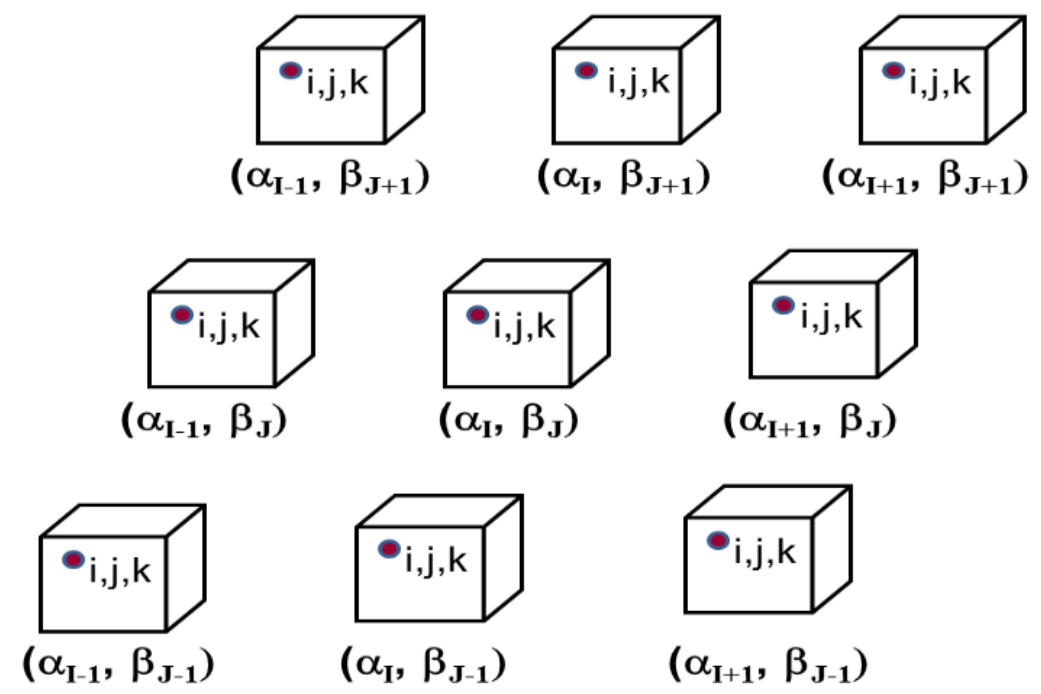

Fig.8 Indexing of Embedded Blocks for Pointwise Block Spectrum

Given a set of embedded fine mesh blocks, a mesh-pointwise spectrum for mesh cell (i, j, k) of block (I, J) is given by a double Fourier series retaining $\mathrm{M}_{\mathrm{h}} \mathrm{X} \mathrm{N}_{\mathrm{h}}$ harmonics,

$$
\begin{aligned}
\left(T S T_{i, j, k}\right)_{I, J}= & \sum_{m=0, n=0}^{M_{h, N_{h}}} \lambda_{m, n}\left[A_{m, n} \cos \left(m \alpha_{I}\right) \cos \left(n \beta_{J}\right)+B_{m, n} \sin \left(m \alpha_{I}\right) \cos \left(n \beta_{J}\right)\right. \\
& \left.+C_{m, n} \cos \left(m \alpha_{I}\right) \sin \left(n \beta_{J}\right)+D_{m, n} \sin \left(m \alpha_{I}\right) \sin \left(n \beta_{J}\right)\right]
\end{aligned}
$$

where: $\quad \lambda_{\mathrm{m}, \mathrm{n}}=\frac{1}{4}, \quad(m=n=0)$

$$
\begin{array}{ll}
\lambda_{\mathrm{m}, \mathrm{n}}=\frac{1}{2}, & (m>0, n=0 \text { or } \quad m=0, n>0) \\
\lambda_{\mathrm{m}, \mathrm{n}}=1, & (m>0, n>0)
\end{array}
$$

For mesh cell (i, j, k), the corresponding Fourier coefficients are obtained from the solutions of $\mathrm{M}_{\mathrm{b}} \mathrm{XN}_{\mathrm{b}}$ embedded blocks by:

$$
\begin{aligned}
& A_{m, n}=\frac{4}{M_{b} N_{b}} \sum_{I=1, J=1}^{M b, N b}\left(T S T_{i, j, k}\right)_{I, J} \cos \left(m \alpha_{I}\right) \cos \left(n \beta_{J}\right) \\
& B_{m, n}=\frac{4}{M_{b} N_{b}} \sum_{I=1, J=1}^{M b, N b}\left(T S T_{i, j, k}\right)_{I, J} \sin \left(m \alpha_{I}\right) \cos \left(n \beta_{J}\right)
\end{aligned}
$$




$$
\begin{aligned}
& C_{m, n}=\frac{4}{M_{b} N_{b}} \sum_{I=1, J=1}^{M b, N b}\left(T S T_{i, j, k}\right)_{I, J} \cos \left(m \alpha_{I}\right) \sin \left(n \beta_{J}\right) \\
& D_{m, n}=\frac{4}{M_{b} N_{b}} \sum_{I=1, J=1}^{M b, N b}\left(T S T_{i, j, k}\right)_{I, J} \sin \left(m \alpha_{I}\right) \sin \left(n \beta_{J}\right)
\end{aligned}
$$

After the Fourier spectrum for mesh cell (i, j, k) is established, all the corresponding mesh cells (i, $\mathrm{j}, \mathrm{k}$ ) of all those blocks in un-embedded regions can be updated (Eq.25) according to the corresponding phase angles in the two directions $\alpha_{\mathrm{I}}, \beta_{\mathrm{J}}$, (Fig.8). It should also be noted that although a full Fourier spectrum would require a spatial periodicity, a non-periodic distribution can be simply regarded as one half of a periodic variation by padding to generate the other imaginary half [8] [10].

With the source terms being mapped from the local fine mesh blocks to the global coarse mesh domain, we can now have a two-way coupling between the two mesh domain solutions. The base mesh solution provides the global environment and equivalent boundary conditions for each and all of the discretely embedded fine mesh domains. The function of the source terms propagated through the block spectra is to ensure that the base mesh flow field in a non-embedded region is equally influenced by an equivalent fine mesh solution locally even though there is no local fine mesh embedded. On the other hand, the fine mesh solutions of those embedded blocks will now be subject to a right surrounding environment (boundary conditions) provided by the global base mesh solution with the propagated source terms, equivalently to that provided by a global solution with the domain fully discretized with the fine mesh resolution, even though the fine mesh resolution is only available in those few discretely embedded blocks.

\section{RESULTS OF BLOCK SPECTRAL SOLUTIONS}

\subsection{Unsteady Mixing of Multiple Jets}

Unsteady interactions among multiple jets are considered in this case. The 2D computational domain of a length $0.3 \mathrm{~m}$ in the streamwise direction is subject to 20 horizontal jets (from the left to right, with a pitch/width ratio of 3), as shown in Fig.9. The background base flow is at a uniform condition of an inlet stagnation pressure 100,000 $\mathrm{Pa}$, and an exit static pressure of 95,000 $\mathrm{Pa}$. Reynolds number based on the streamwise domain length is $10^{5}$. The jet inlet stagnation pressure is subject to a pitchwise distortion in a form of: 


$$
P_{0 j}=\overline{P_{0 j}}\left(1-A_{p 0 j} \cos \theta_{j}\right) \quad\left(\theta_{j}=\frac{2 \pi j}{20}, \quad j=1,2, \ldots, 20\right)
$$

where the mean stagnation pressure for the 20 jets is $105000 \mathrm{~Pa}$ and the amplitude of the distortion is $20 \%$ of the dynamic head.

The base coarse mesh has 201 (streamwise) x 491(pitchwise) mesh cells. The fine mesh domains have 5x5 embedded mesh cells for each coarse mesh cell. An instantaneous flow field in terms of entropy contours is shown in Fig. 10 for the direct fine mesh solution, where the fine mesh covers the whole domain. The jets are largely steady up to $30 \%$ of the domain length. Afterwards, the self-excited unstable shear layer patterns start to emerge and develop, initially for each of the jets on its own. These unsteady patterns of the jets grow rapidly from $50 \%$ domain length onwards. Each jet interacts strongly with its neighboring ones, and adjacent jets are highly entrained to each other when they reach the domain exit.

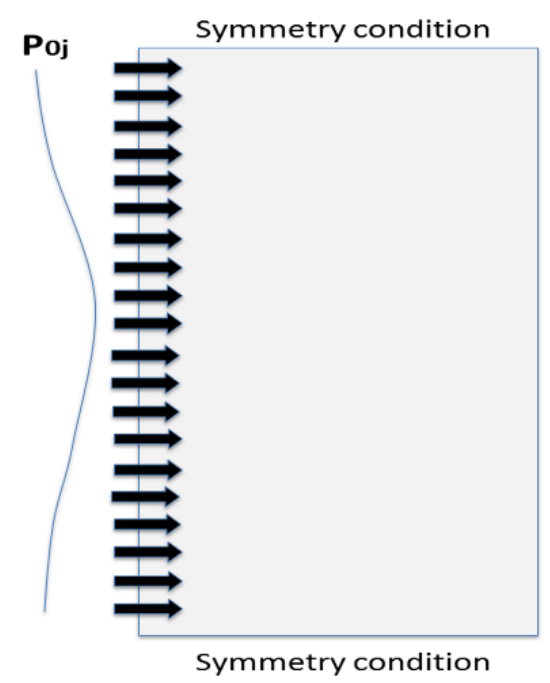

Fig.9 Computational Domain for 20 Jets Subject to Inlet Total Pressure Distortion 

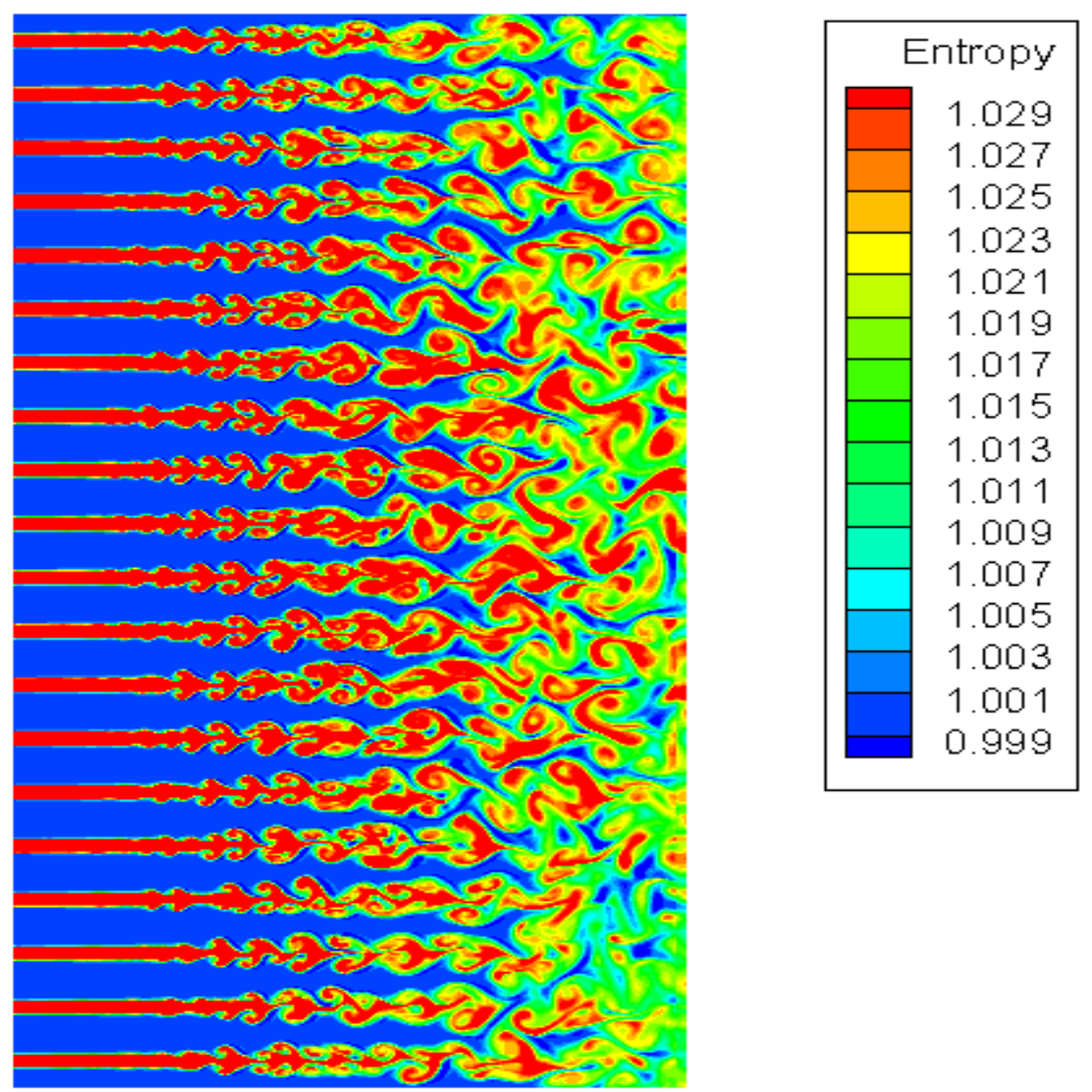

Fig.10 Instantaneous Entropy Contours (Direct Fine-mesh Solution)

Before we proceed with analyzing the flow solutions with the new block spectral method, the mesh-dependency of the direct solutions is first examined. Fig.11 shows the time-averaged streamwise (x) velocity distributions in the normal (y) direction at the plane $90 \%$ domain length. The y distance is normalized by the jet-jet interval (pitch) w. The solution of the base coarse mesh is compared with the 3 fine mesh solutions with 3x3, 5x5 and 7x7 mesh cells embedded in each base mesh cell respectively. The base mesh solution is shown to be completely steady due to its poor spatial resolution and consequent inability in resolving the self-excited shear layer instabilities. The solution of the 3x3 embedded mesh, though qualitatively comparable to the finer mesh solutions, still indicates noticeable mesh dependency. On the other hand, the differences in the solutions between the $5 \times 5$ and $7 \times 7$ embedded meshes are much smaller. The agreement between the two fine meshes gives a clear indication of mesh convergence. Hence the fine mesh blocks with 5x5 fine mesh cells embedded in each base mesh cell are used for the further analyses. 


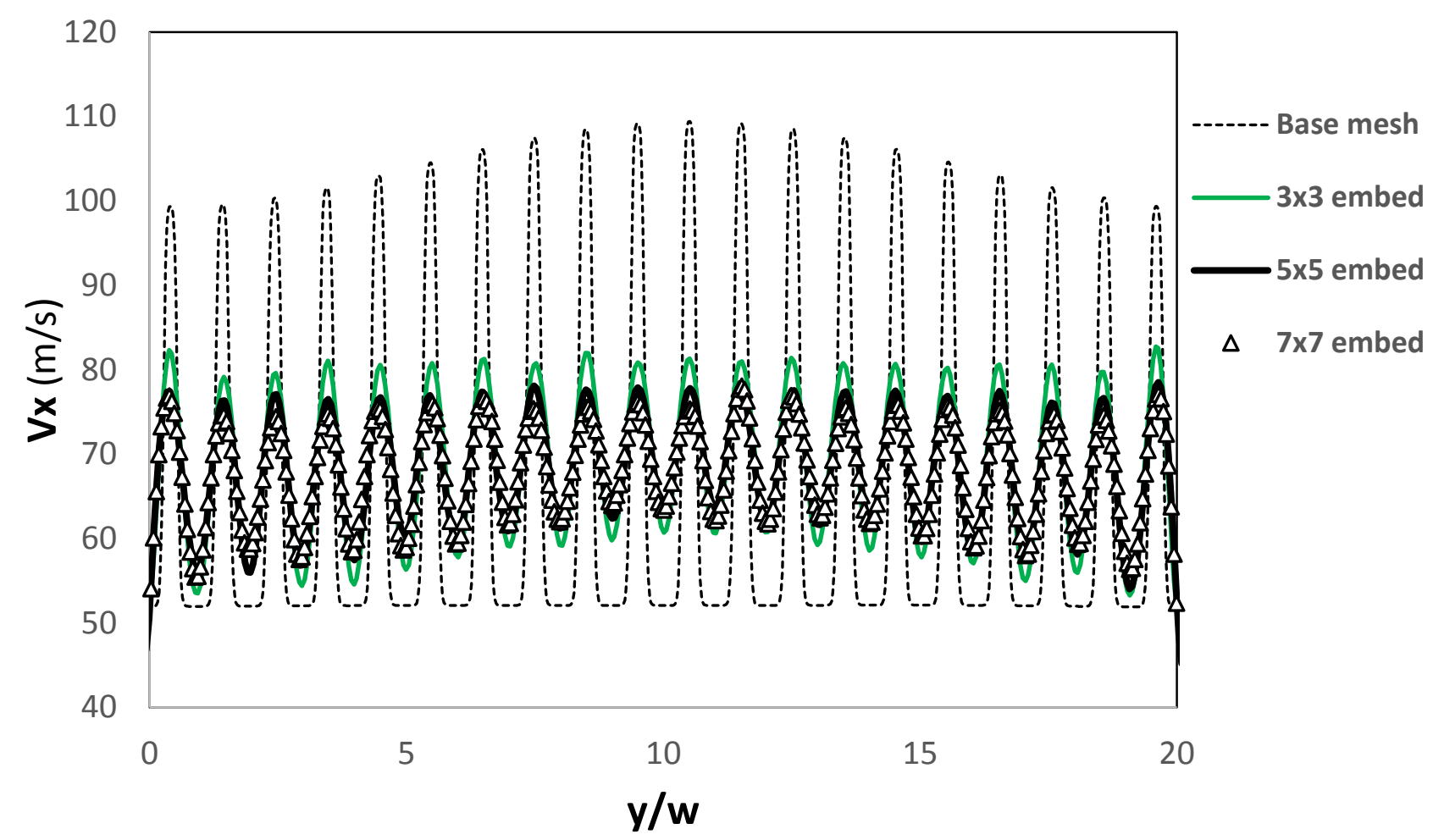

Fig.11 Mesh Dependency Characteristics of Time-averaged Velocities (at 90\% domain length)

For the multi-scale block spectral method, we would like to solve a much smaller number of jets with the corresponding small number of embedded fine mesh blocks while still getting an equivalent fine mesh solution for the whole domain. If the pitchwise block-block spectral representation contains one harmonic, then only 3 blocks will be needed. In this case, one can have each local embedded domain containing only one jet, and apply the periodic condition for the corresponding fluctuation components (Fig.12a). This however will truncate some interactions between adjacent jets. One can also include two jets in one block, and map the source terms from the middle regions between the two jets (Fig.12b). Or, each fine mesh block can contain 3 jets and we can map the source terms from the region of the middle jet (Fig.12c). 


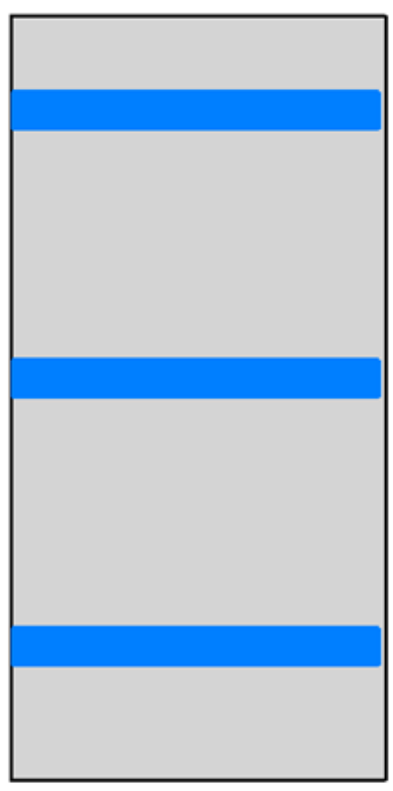

(a) 1 Jet / Block

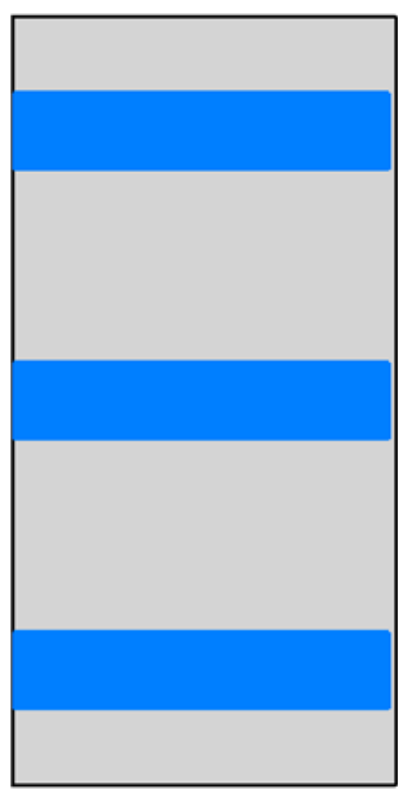

(b) 2 Jets / Block

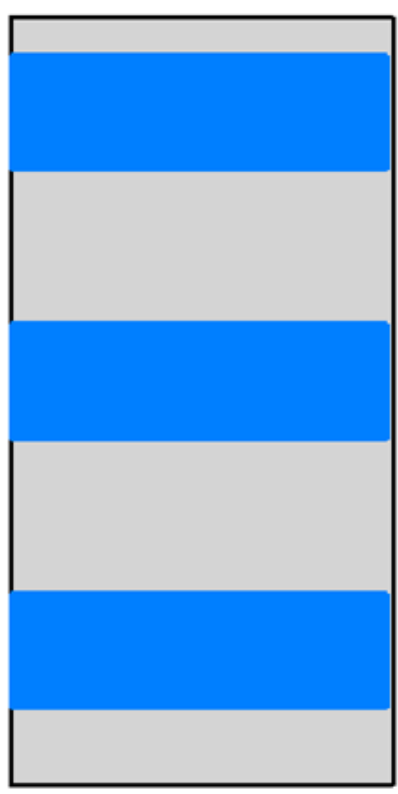

(c) 3 Jets / Block

Fig.12 Embedded Fine Mesh Blocks (in Blue) with Different Numbers of Jets for Each Block

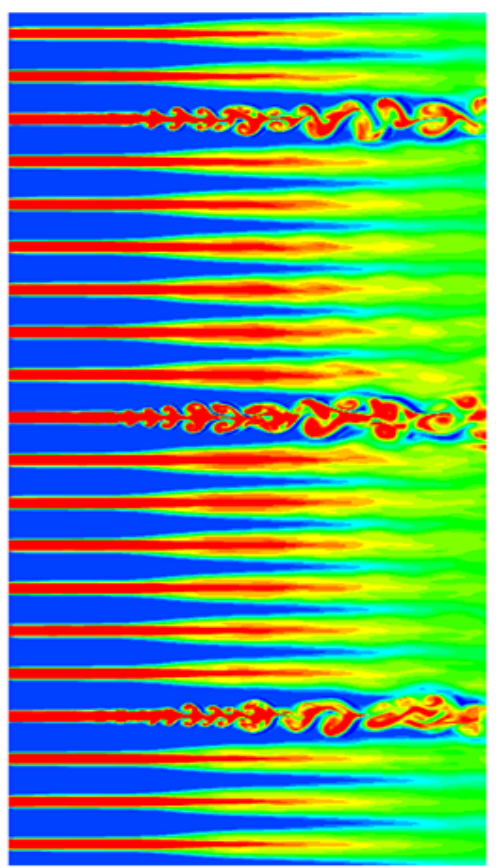

(a) 1-jet Block

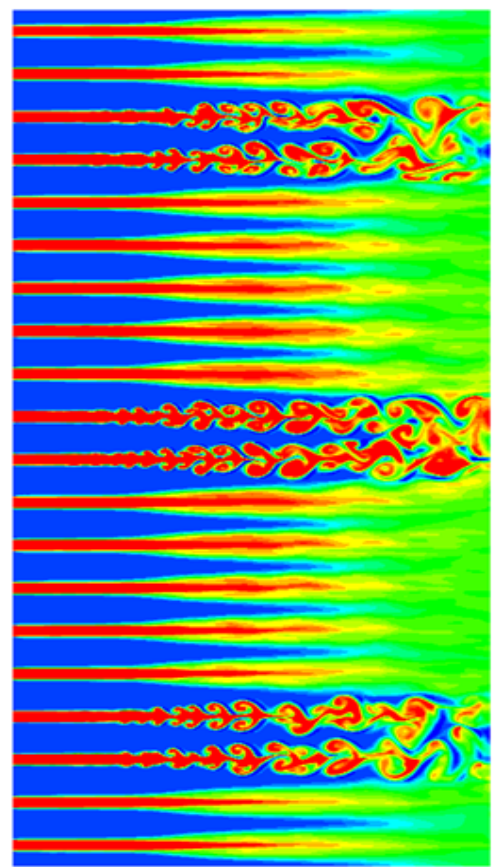

(b) 2-jet Block

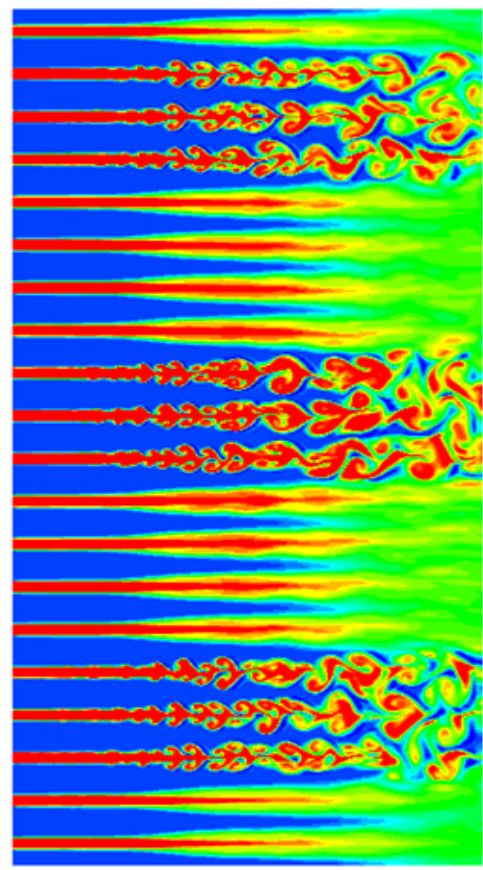

(c) 3-jet Block

Fig.13 Instantaneous Entropy Contours (Block spectral solutions with 3 embedded blocks) 
Fig.13 shows the instantaneous entropy contours of the three cases with different numbers of jets in each fine mesh block. These contour plots already indicate that the unsteady mixing of the jets is shown to be weaker for the 1-jet block (Fig.13a), compared to that with 2 jets (Fig.13b) and 3 jets (Fig.13c) in each embedded fine mesh block respectively. There also appears to be a smaller difference in the mixing between the 2-jet blocking and 3-jet blocking. The corresponding timeaveraged streamwise velocity contours of the block spectral solutions are compared to the direct solution in Fig.14. Clearly reflected in the time-averaged velocities is the under-predicted unsteady mixing if only one jet is included in each embedded block (Fig.13a, Fig.14a). The embedded domains with 2-jets/block (Fig.14b) clearly improve the results and the solution is in good agreement with the direct fine mesh solution (Fig.14c).

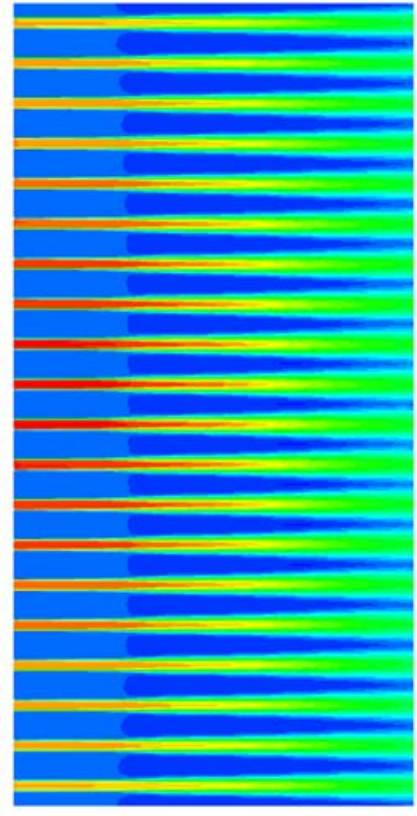

(a) 1-jet Block

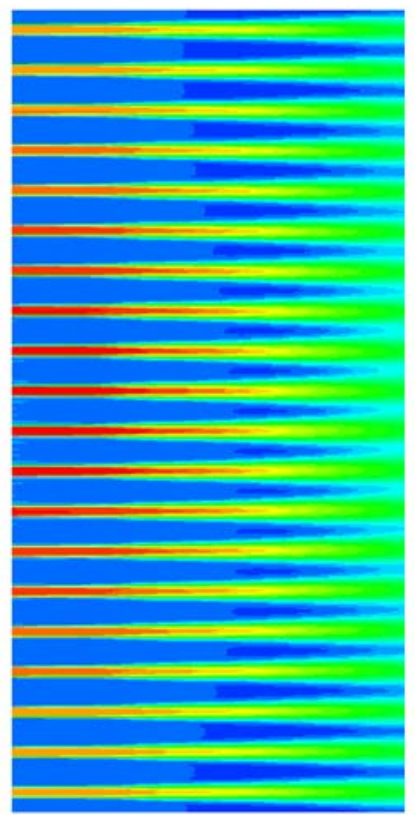

(b) 2-jet Block
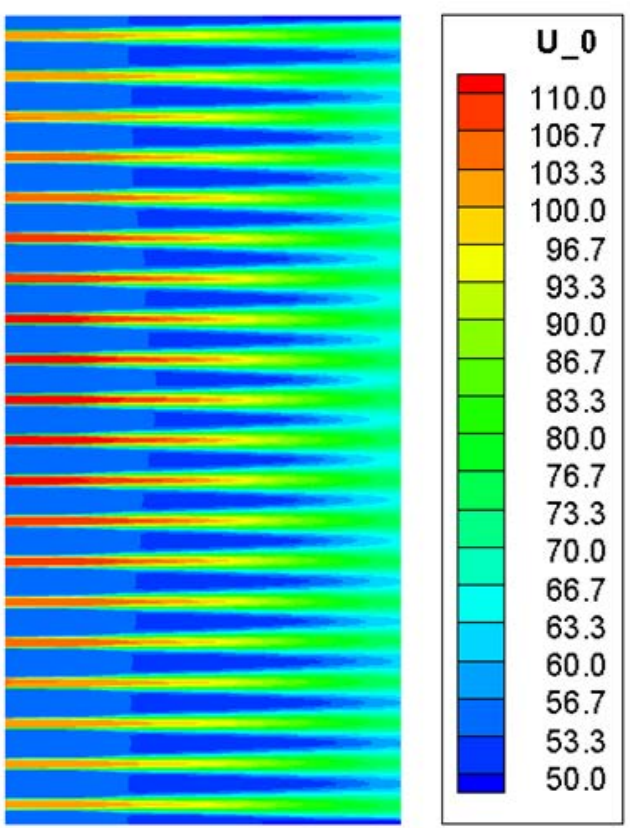

(c) Direct Solution

Fig.14 Time averaged x velocity contours (Block-spectral vs Direct)

A more quantitative examination is made on the time-averaged axial velocity distributions in y direction on a plane at $90 \%$ domain length, as shown in Fig.15. In addition to the time averaged velocities of the 3 cases with different numbers of jets contained in each fine mesh blocks, a RANS solution with the k- $\omega$ SST model closure [20] on the base mesh is also included to highlight the inadequacy of the RANS for this kind of unsteady flows. The solution of one-jet for each fine 
mesh block (Fig.14a) is shown to under-predict the unsteady mixing effect considerably, consistent with the observation on the corresponding flow field (Fig.13a). A clear improvement is achieved when the strong jet-jet interaction is captured by just including 2 jets in each embedded fine mesh block. The solution of 2 jets for each fine mesh block is shown to agree well with the direct fine mesh solution. These results suggest that for this kind of the multi-scale unsteady problems, the unsteady interactions among micro-structures are dominated by those between adjacent ones, thus underpinning the applicability of the present source term based multi-scale block-spectral method with truncated local fine mesh blocks.

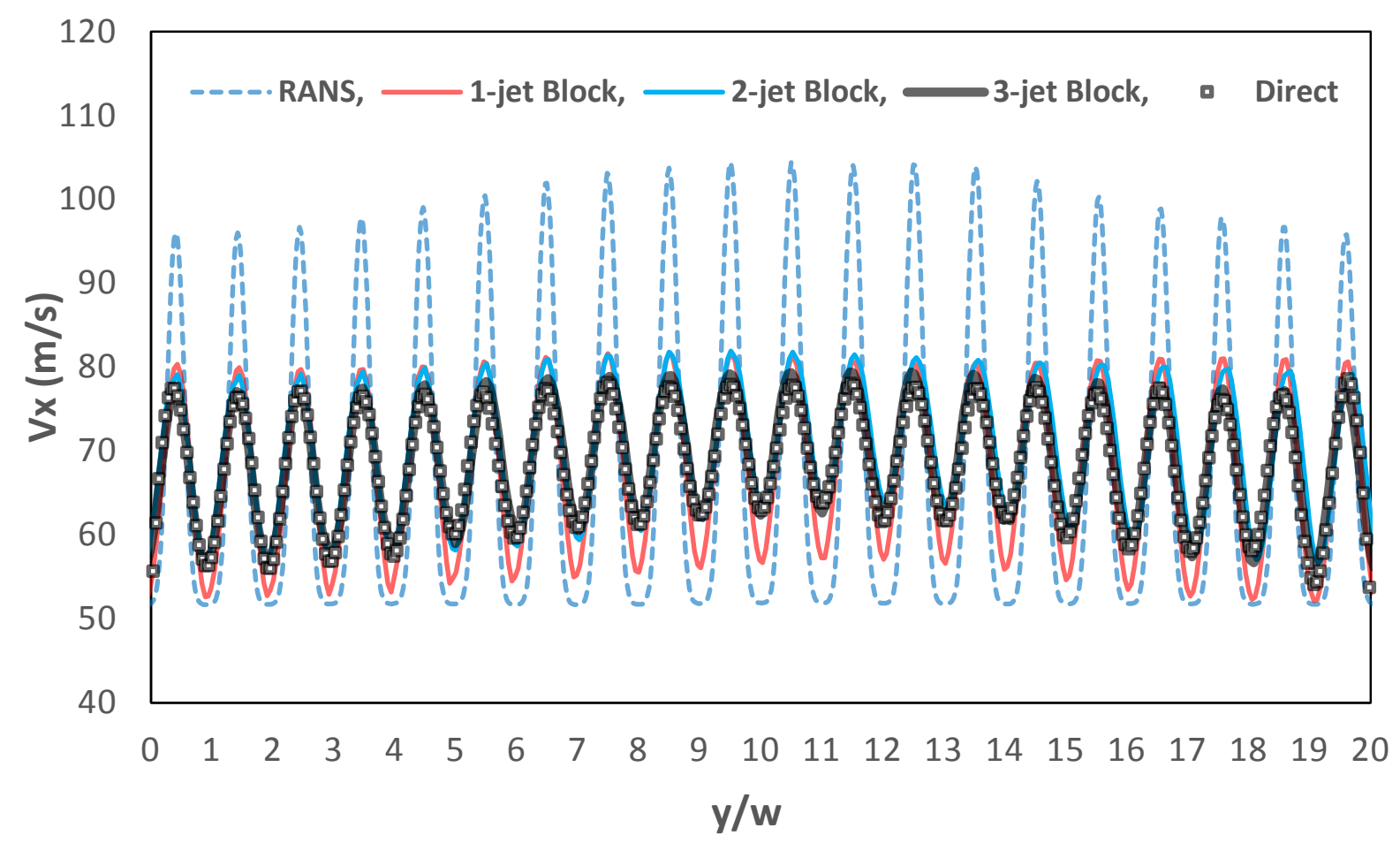

Fig.15 Time-averaged x Velocity Distributions in y at 90\% Domain Length

\subsection{Flow through Multi-row Cylinders}

For more general situations, we are interested in having embedded blocks discretely in the streamwise direction. Now we consider a case for which the feasibility to embed discrete fine mesh blocks in the streamwise direction is examined. The extra issue we have to deal with is the upstream influence on the discretely embedded blocks.

A case of a flow through multiple rows of cylinders is tested here. The domain consists of 34 rows, each row of 3 cylindrical pins, thus 102 cylinder pins in total, as shown in Fig.16. The inflow 
is supplied with a stagnation pressure of $100,000 \mathrm{~Pa}$. The exit static pressure is specified to be 85,000 $\mathrm{Pa}$. The top and bottom boundaries are subject to the symmetry (inviscid) boundary condition. The Reynolds number based on the cylinder diameter and the inlet velocity is taken to be 1000 .

Symmetry condition

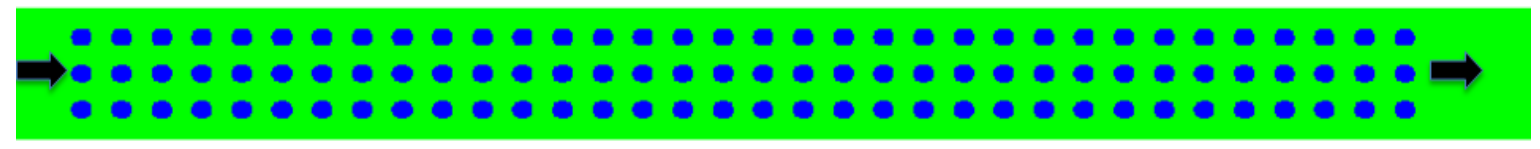

Symmetry condition

Fig.16 Computational Domain and Boundary Conditions for 34x3 Cylinders

There are two kinds of upstream influences to be dealt with for multi-scale problems. The first is the inlet flow influence with a very strong history effect. For the present case, we can see that the first few rows of the cylinders will be subjected to the strong influence of the under-developed flow, as shown by the instantaneous unsteady flow and the time-averaged flow field (Fig.17). In terms of the time-averaged flow (Fig.17b), it takes about 5-6 rows for the flow to develop into a similar rowrow pattern. For this inlet region, we should only consider the domain truncation in the direction normal to the streamwise one, as for the jet mixing example presented in 5.1. On the other hand, for the bulk flow region in the middle of the domain, the time-averaged flow shows a remarkably similar pattern from one row to the next (Fig.18b), even though the corresponding instantaneous unsteady flow does not seem to indicate any row-row similarity (Fig.18a). 


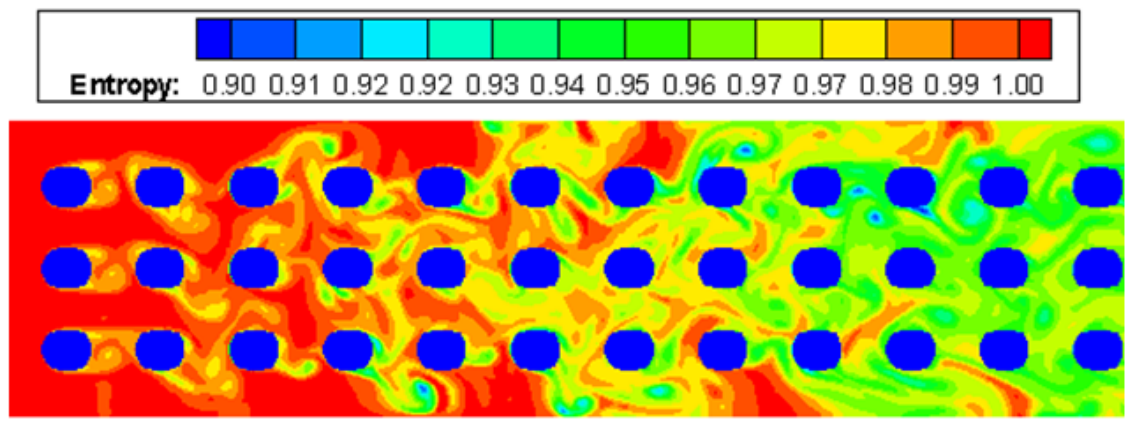

(a) Unsteady Entropy

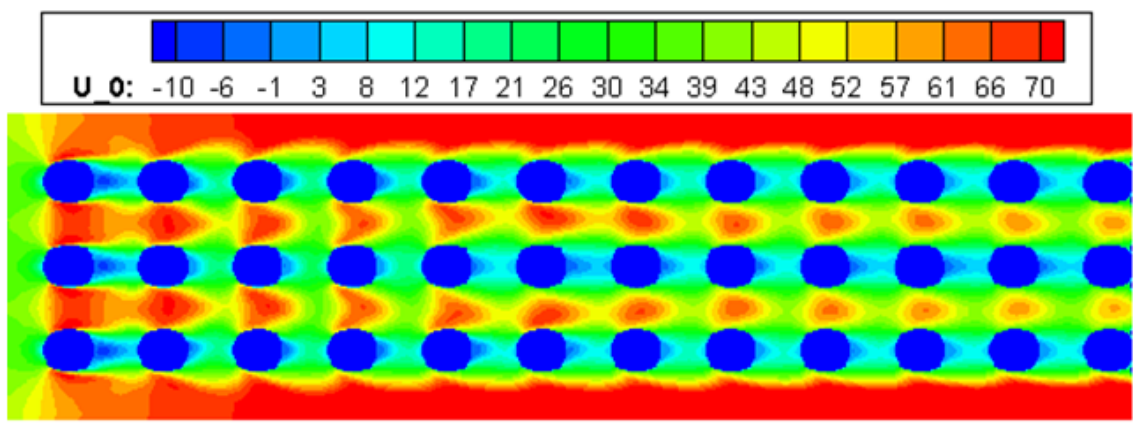

(b) Time-averaged Velocity

Fig.17 Local Flow Field with Strong Upstream Influence (Inlet Region)

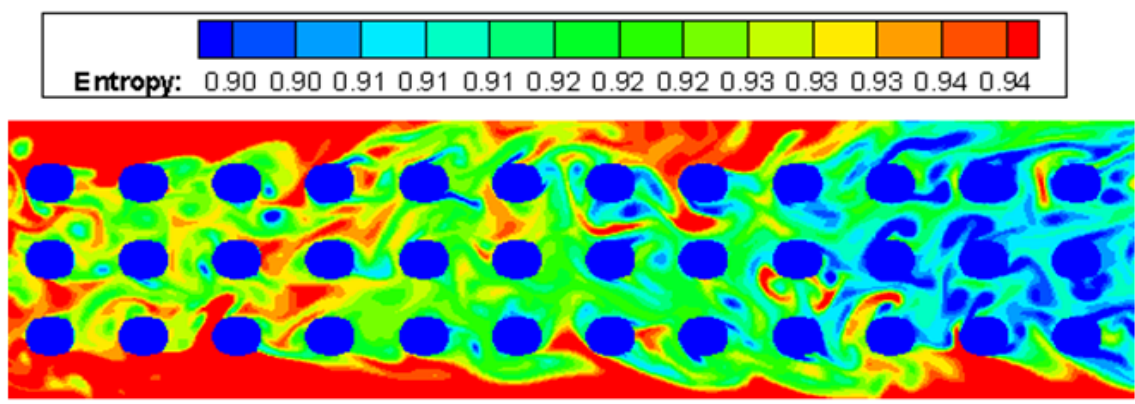

(a) Unsteady Entropy
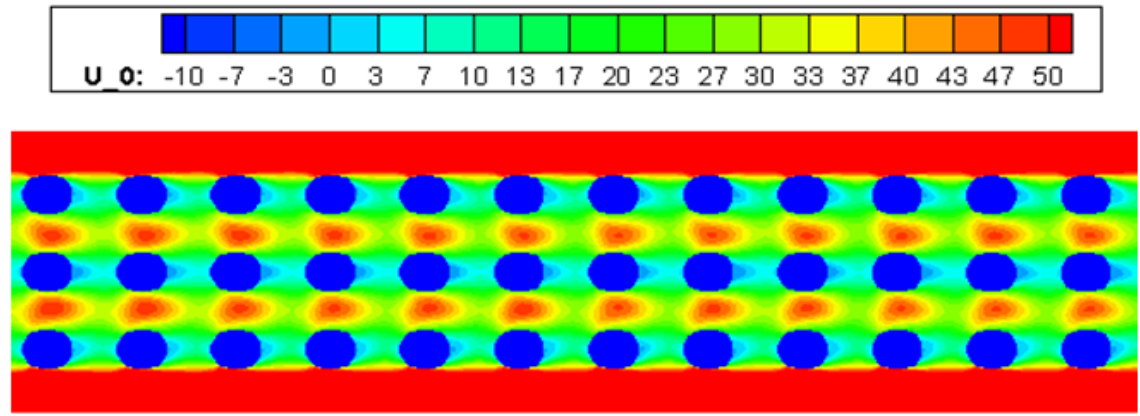

(b) Time-averaged Velocity

Fig.18 Flow Field with Similar Row-Row Pattern (Bulk Flow Region) 
For a direct solution, all 34 rows of cylindrical pins are solved with a fine mesh embedded on a base mesh. In this case, a coarse base mesh of 290 (streamwise) x 31(normal) is taken for the whole domain. The base mesh is very coarse, and each cylinder circumference is covered by only 12 mesh cells. $5 \times 5$ fine mesh cells are embedded in each coarse mesh cell. Thus, for the embedded fine mesh, the cylinder circumference will be resolved by 60 mesh points. The nonslip viscous wall condition is applied to the solid wall surfaces. For a coarse mesh, a nonslip wall condition is only applicable when the source terms are applied.

Consider the embedded blocks for the block spectral mapping. Given the strong upstream effects in the inlet regions, the first 6 rows of the domain remain fully embedded with the fine mesh without any domain truncation as in the direct solution. Similarly, the last row (row 34) remains embedded with the fine mesh to provide the bulk region with a similar downstream condition. The region between row 7 and row 33 is treated as the bulk flow region to which discrete fine mesh blocks are embedded and the block spectral mapping is applied.

For each embedded fine mesh block, we need to further consider its own inflow boundary. As introduced in the method section (4.1), an inflow boundary is updated from two sources. The surrounding coarse base mesh points will provide the base flow variables by a $2^{\text {nd }}$ order accurate interpolation. On top of this base mesh flow part, additional non-uniform disturbances subject to the fine mesh resolution will be taken from the corresponding points of a downstream boundary of the block. This simultaneous updating will provide inflow small scale disturbances, as if the embedded fine mesh block immediately followed an upstream fine mesh block. This inflow treatment can be regarded as a simple form of the 'recycling' inflow turbulence generation, similar to that as applied to LES in a truncated domain (e.g. Lund et al [26]).

The computations are carried out by embedding the bulk region with different number of rows. The sensitivity to the upstream condition is examined by comparing the results between solutions of having different numbers of rows of cylinders in each embedded fine mesh block. When the fine mesh block contains only one row of cylinders, the source terms have to be collected from this row itself for setting up the block-to-block spectrum. When the fine mesh block contains two rows of cylinders, the source terms are collected from the second row for having a more realistically established upstream influence for the row and thus minimizing the influence of the recycling condition at this inflow boundary of the block. 
The time-averaged flows for the bulk region are compared between the direct solution and the block-spectral solutions with 1 row, 2 rows and 3 rows of cylinders contained in each embedded block respectively, as shown in Fig.19. The corresponding time-averaged $\mathrm{x}$ velocity profiles downstream of the last cylinder row are compared in Fig.20. It can be seen that the solution with single-row for each embedded block does not agree well with the direct solution and those block spectral solutions with 2 rows or 3 rows of cylinders in each embedded block respectively. This suggests that the simple recycling inflow condition still has a non-negligible erroneous influence on the first row. The influence of the inflow condition on the subsequent $2^{\text {nd }}$ and $3^{\text {rd }}$ row appears to be however significantly weaker, thus both solutions with multi-rows (even just 2 rows) of cylinders in each embedded block agree well with the direct solution.

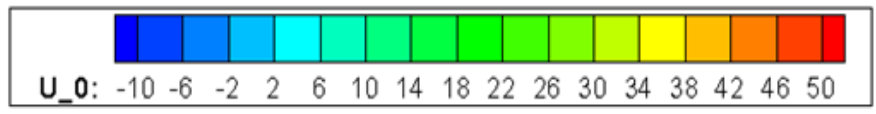

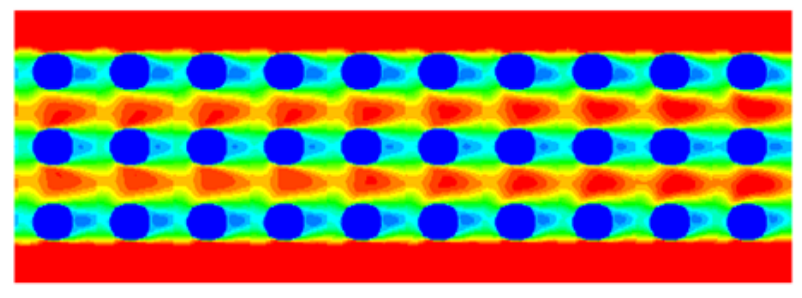

(a) 1 Row / Embedded Block

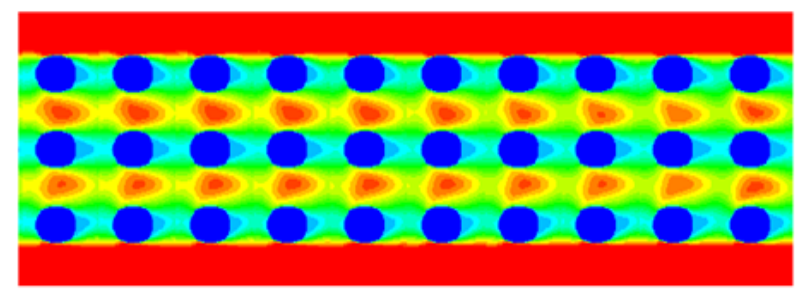

(c) 3 Rows/Embedded Block

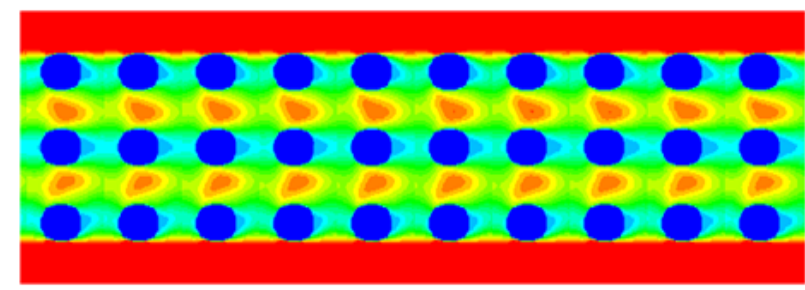

(b) 2 Rows / Embedded Block

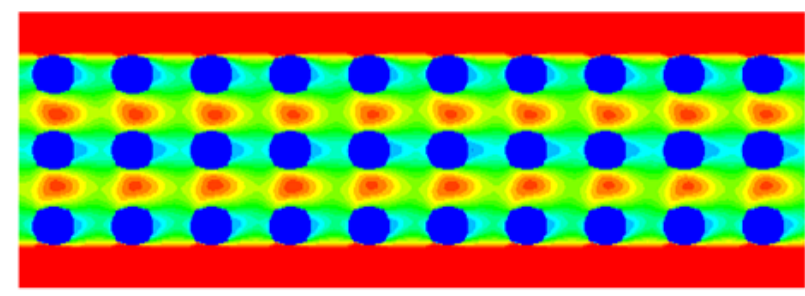

(c) Direct Solution

Fig.19 Time-averaged Streamwise Velocity Field (Bulk Region)

The results of the mesh dependency characteristics are also examined as shown in Fig.21. Here the time averaged velocity profiles are checked for three mesh densities, 3x3 (coarse), $5 \times 5$ (nominal) and 7x7 (fine) embedded fine mesh cells for each base mesh cell. Both the direct solutions and the block spectral solutions with the three embedded mesh densities are included. While the mesh dependency is noticeable as indicated by the results with the $3 \times 3$ embedding, the mesh convergence trend is clearly illustrated by the much smaller differences between the solutions with the $5 \times 5$ and 
7x7 embedded fine mesh cells. It is also of interest to note the good agreement between the direct solutions and corresponding block spectral solutions for all 3 mesh densities respectively. Thus not only do the block spectral results agree well with those of the direct solutions when the solutions become largely mesh independent as required, the present block spectral method also seems to predict the same trend of the mesh-dependence as the direct method does as well.

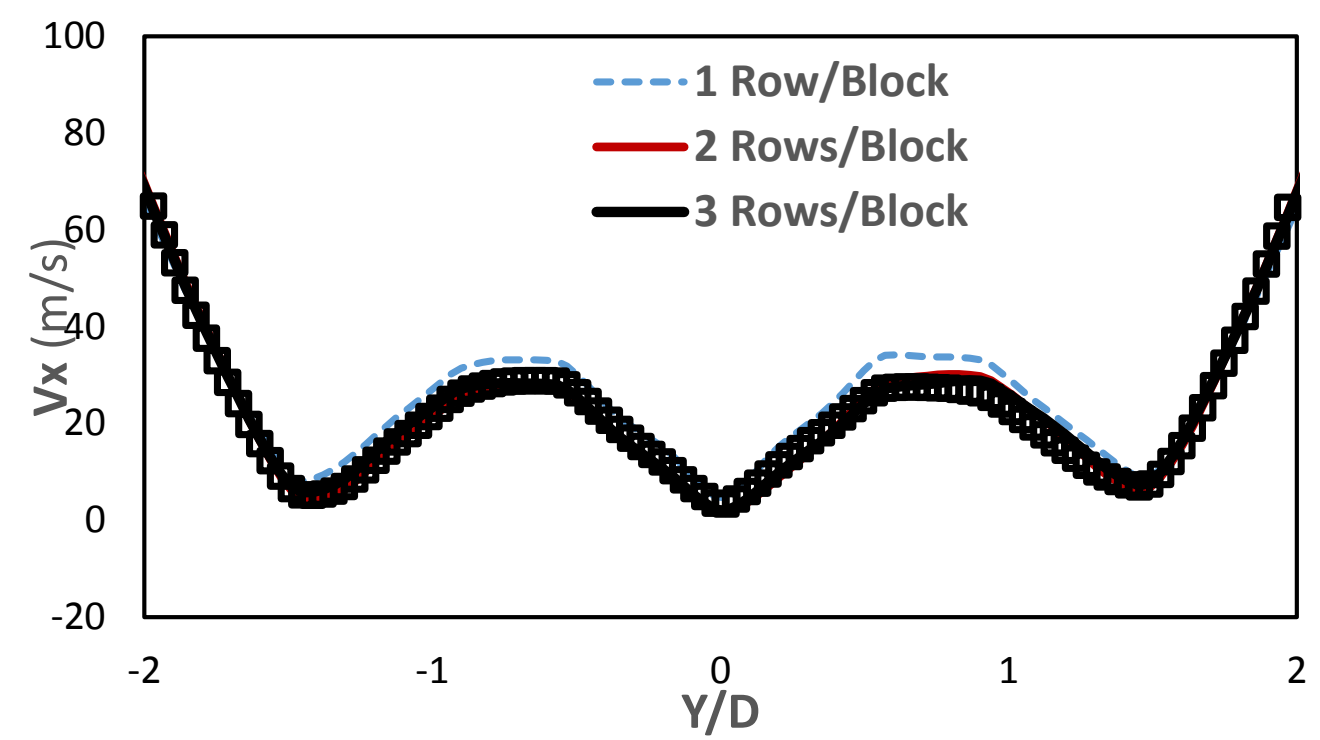

Fig.20 Time-averaged Streamwise Velocities Downstream of Last Row of Cylinders

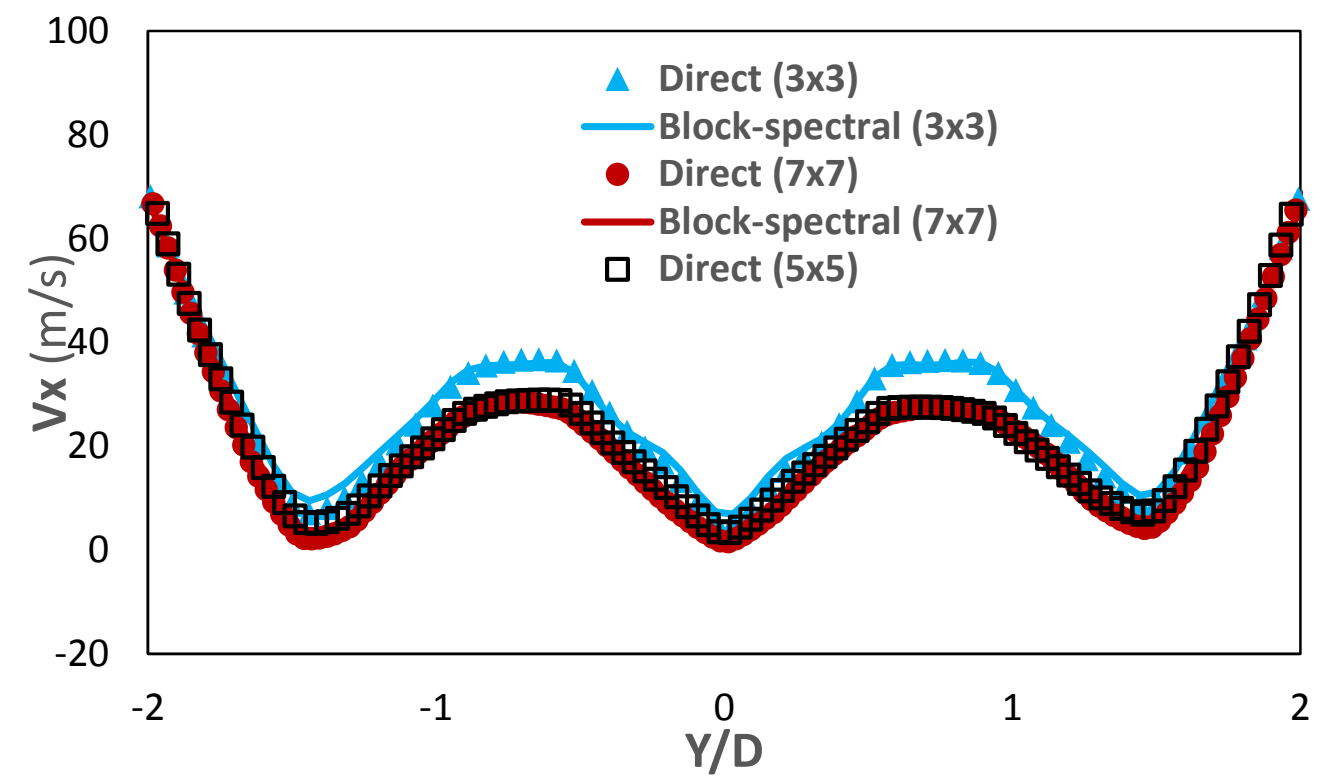

Fig.21 Time-averaged Velocities Downstream of Last Row (mesh dependency characteristics) 


\subsection{Multi-hole Effusion Cooling}

The final test case is set up particularly to examine and demonstrate the capability of the present method for large eddy simulations (LES). For this purpose, a 3D flow over a flat-plate subject to coolant injections through multiple holes of a small size is considered.

The computational domain is shown in Fig.22. The flat plate is of a length of $0.03 \mathrm{~m}$, and a width (in the lateral direction) of $0.15 \mathrm{~m}$. The main stream flow is parallel to the plate at the inflow condition of a stagnation temperature of 300K and an average stagnation pressure of 100,000 Pa. The exit condition is specified as an exit static pressure of 98,000 Pa. The inflow is also subject to a sinusoidal total pressure distortion in the lateral direction in the same form as given in Eq.27. The amplitude of the inlet distortion is $50 \%$ of the mean dynamic head. The Reynolds number based on the plate length is $5 \times 10^{4}$.

Coolant injection is arranged in a way similar to an effusion cooling, where a very large number of closely spaced small cooling holes are often adopted. In the present case, 15 rows of cooling holes are placed along the lateral direction. Each row has 3 cooling holes, thus there are 45 holes in total. The cooling holes are of the same cylindrical-like geometry and are aligned with the streamwise direction but angled with the plate at $24^{\circ}$ (Fig.22). The coolant is supplied at a stagnation pressure of $102,000 \mathrm{~Pa}$ and a stagnation temperature of $200 \mathrm{~K}$ (thus, the coolant-main stream temperature ratio is 0.666 ).

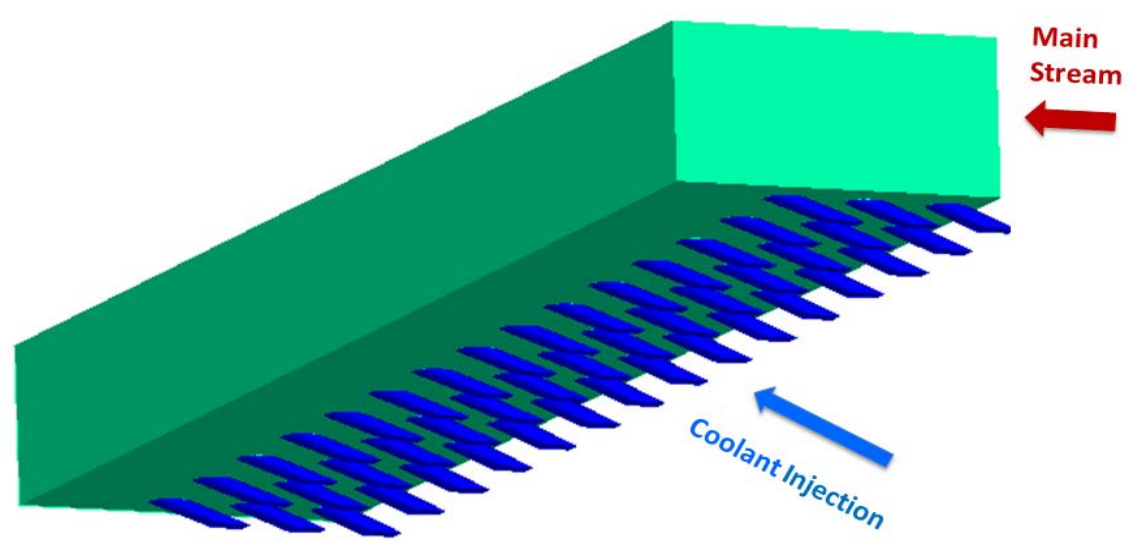

Fig.22 Computational Domain for Effusion Cooling

The base-mesh for the main stream domain has a mesh density of 101x751x31 in the streamwise, the lateral and the plate normal directions. The cooling holes are discretized by a relatively coarse 
mesh of 11x11x21 points for each hole, equivalently with the circumference of the cooling hole being resolved by 40 mesh cells. Even when the coolant supply is laminar, the mixing between the coolant and the main stream is likely to develop to a turbulent flow. It is the turbulent mixing in this region which is the key area of the interest and a challenge for the effusion cooling CFD analysis. In the present case, the bulk of the main stream domain has been embedded by $3 \times 3 \times 3$ fine mesh cells for each base-mesh cell. For a direct solution with the embedded fine mesh, the total mesh count is 54.5 million.

As in the previous test cases, the calculations are carried out without any turbulence model. The mesh resolution capability in capturing and resolving large scale turbulence eddies can be indicated by the results of Fig.23, where the iso-surfaces of total temperature at $T_{0} / T_{01}=0.8$ ( $T_{01}$ is the main stream inlet stagnation temperature) are shown. The total temperature is chosen here, partly because of its relevance to heat transfer and cooling, it is also a largely convective property and thus indicative of the length scales of the flow disturbances resolved. The base mesh solution (Fig.23a) is shown to be largely steady and the tendency of the coolant jet being lifted off the plate in line with a typical counter-rotating vortical flow structure for cooling injection is also indicated.

The direct solution with the full embedded fine mesh (Fig.23b), however indicates that the embedded fine mesh is able to capture and resolve large scale turbulence eddies to a considerable extent. The question of the interest then is how well the present block-spectral method can capture the unsteadiness in discrete local block domains, and propagate the source terms to get the timeaveraged flow for a global domain. Fig. 23c shows the corresponding iso-surface of instantaneous total temperature for the block spectral solution. In this case, three fine mesh blocks are embedded locally for rows 2 and 3 ('r2' and 'r3'), rows 7 and 8 ('r7' and 'r8') and rows 12 and 13 ('r12' and 'r13') respectively as indicated in Fig.23c. The choice of a local embedded block containing two rows of the cooling holes is made based on the previous test case regarding the jet-to-jet interaction as discussed in 5.1. It can be seen that the embedded local mesh blocks do provide a good resolution in capturing large scale turbulence eddies. It is also noted that the flow in the nonembedded regions do show some unsteadiness in contrast to that of the base-mesh solution (Fig.23a). This observation also supports the augmented formulation of the total source terms (Eq.24), in which the unsteadiness of the base-mesh solution is taken into account. 

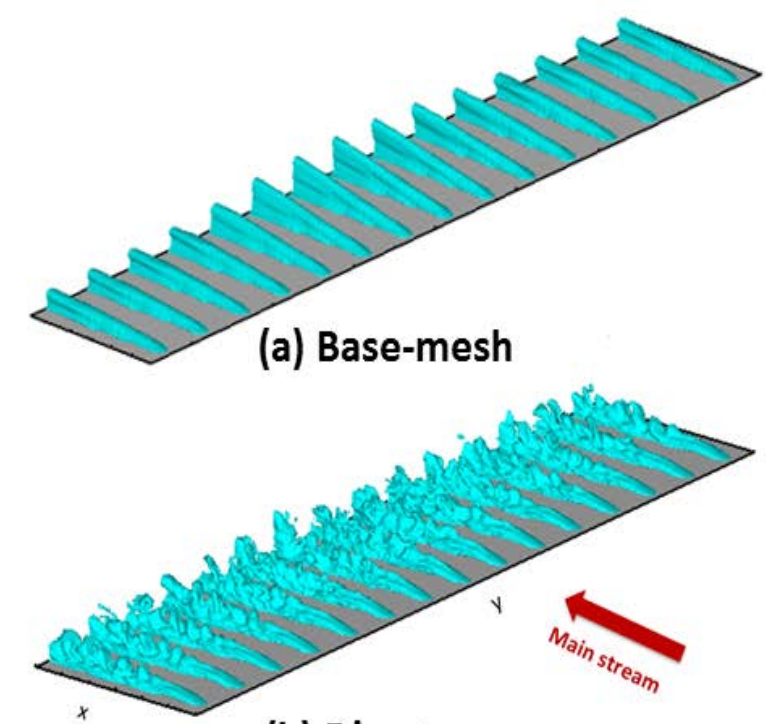

(b) Direct

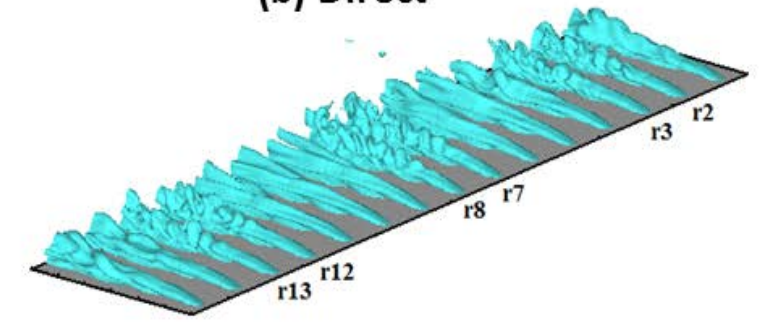

(c) Block Spectral

Fig.23 Iso-surface of Instantaneous Total Temperature $\left(\mathrm{T}_{0} / \mathrm{T}_{01}=0.8\right)$

Now we examine the time averaged flow. The time-averaged total temperature contours on the plate are compared among the base-mesh solution, the fully embedded direct solution and the locally embedded block spectral solution as shown in Fig.24. The base mesh solution is largely steady (as shown in Fig.23a) and the tendency of jet lift-off gives relatively high surface temperatures (lower cooling effectiveness) in the regions downstream of the injection holes (Fig.24a). On the other hand, the embedded fine mesh results for both the fully embedded direct solution (Fig.24b) and the locally embedded block spectral solution (Fig.24c) give generally lower temperatures, which is attributed to the enhanced mixing due to the resolved turbulence, and thus a much reduced tendency for the coolant jets to lift off. It is also interesting to note that the complex coolant-main stream interactions captured by the fine mesh solution give an almost opposite lateral variation (Fig.24b, c) near the domain exit, compared to that of the base-mesh solution (Fig.24a). The surface temperature distributions along the lateral distance direction at 75\% domain length, downstream of the cooling 
holes are plotted in Fig.25. The lateral distance in y is normalized here by the hole-to-hole distance in the lateral direction $\mathrm{w}_{\mathrm{h}}$. We can see that the base-mesh solution produces notably high temperatures, particularly downstream of those cooling holes near the upper and lower ends of the domain. On the other, the block spectral method is shown to be able to produce lower surface temperatures in these regions in good agreement with the direct solution.

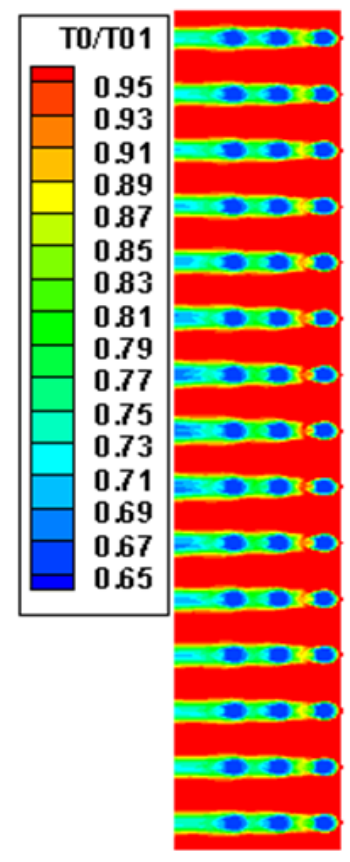

(a) Base-mesh

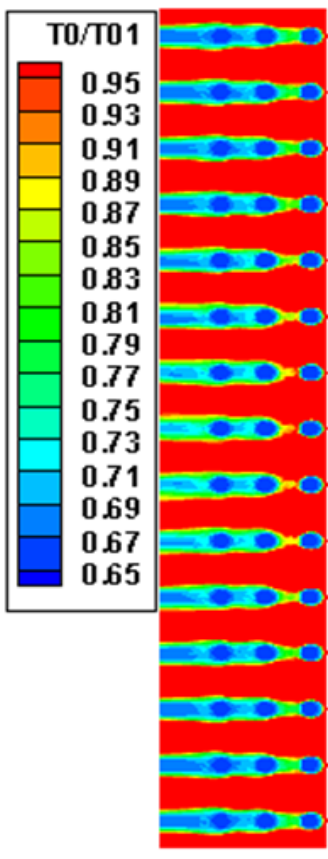

(b) Direct

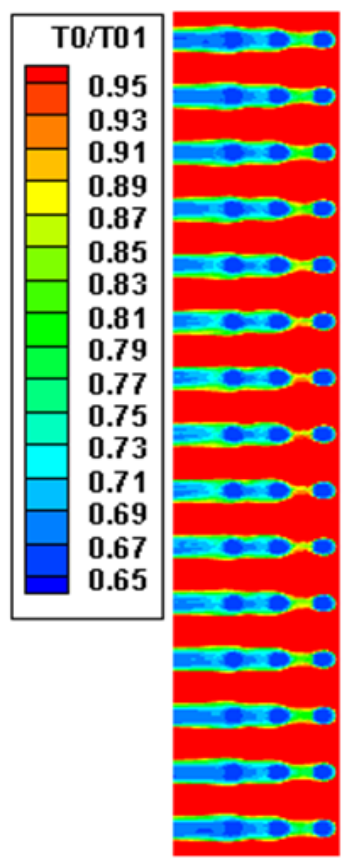

(c) Block Spectral

Fig.24 Time-averaged surface temperature contours (inflow from the right)

Overall, the computational studies of these test cases consistently demonstrate the validity of the present procedure and implementation, and the good agreement between the present solutions and the corresponding direct solutions serves clearly as a concept proof of the proposed multi-scale block spectral methodology for unsteady flows. 


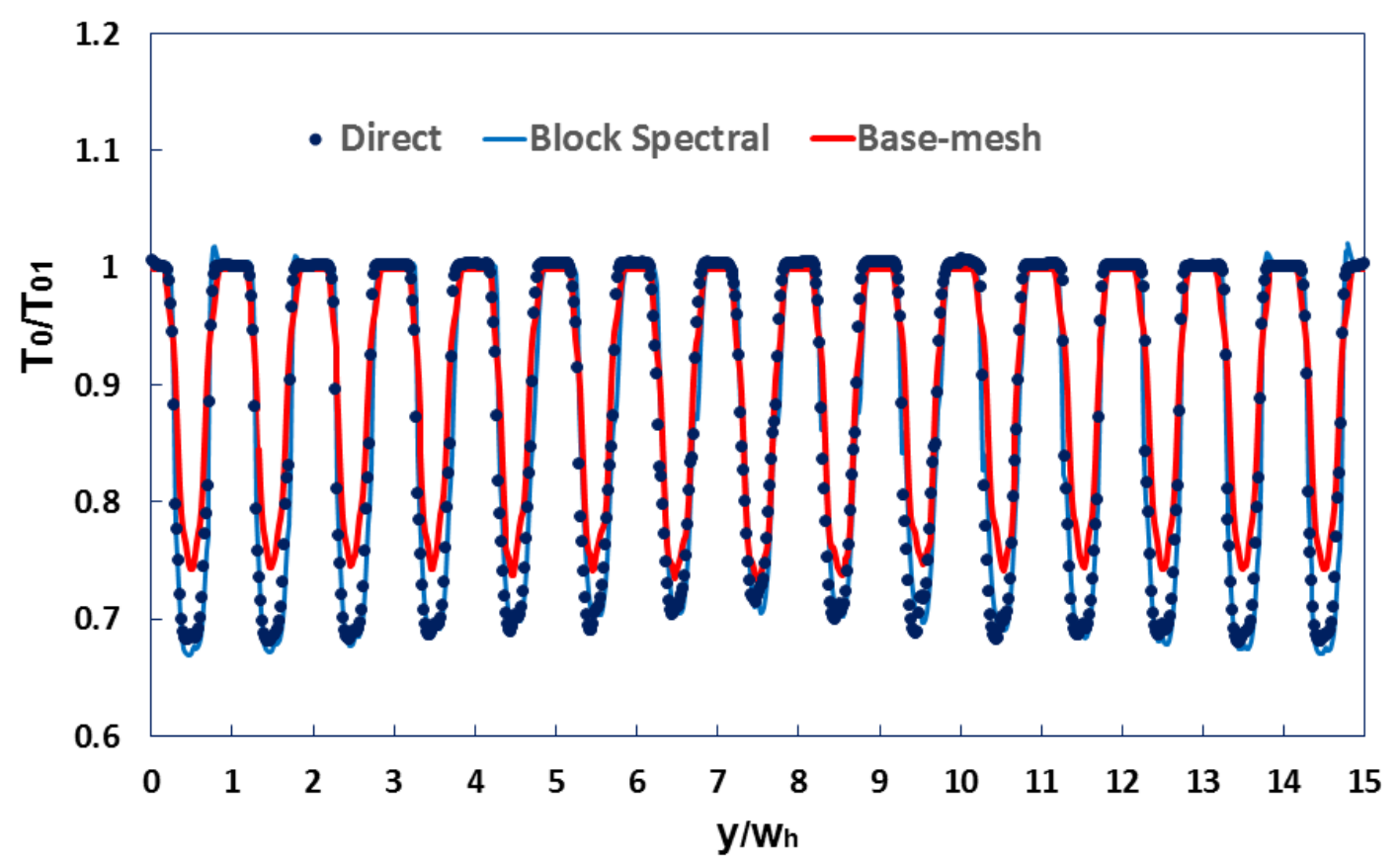

Fig.25 Time-averaged surface temperature distributions along lateral distance (at $75 \%$ domain length)

\section{CONCLUDING REMARKS}

One of the main challenges in computational fluid dynamics is to deal with problems with distinctive and disparate scales and a huge multiplicity of similar small scale structures. There is an increasing interest in resolving (instead of modelling) the small scale structures and their impact on performance parameters of practical interest, in particular in the context of surface manufacturable regular micro-structures. A block spectral approach is adopted to make use of the corresponding scale-dependent solvability. The global domain is decomposed into a large number of similar small block domains. The block spectra link the small blocks so that only a small set of fine mesh blocks need to be embedded and solved with a high mesh resolution. The whole domain solution can thus be very efficiently obtained by a block spectral mapping. The previous block spectral method has been only developed for steady and quasi-steady flow problems. This is restrictive in general. For the micro-structures of interest in particular, there tends to be self-excited unsteadiness due to typical bluff body shapes of micro-elements. The present work extends the 
block spectral method to unsteady flows of short temporal and spatial scales (e.g. those due to selfexcited unsteady vortices and turbulence disturbances). The overall approach stems from the observed similarity among micro-structures for the time-averaged flow, to which the block spectral mapping is justifiably applicable. The interaction between the global coarse mesh and local fine mesh domains is enabled by a source term approach. Source terms are generated from the local fine mesh blocks and propagated to the global coarse mesh domain by the block spectral mapping. Consequently, the global coarse mesh solution provides a right environment and boundary conditions for the local fine mesh blocks, whilst the local mesh solutions provide the propagatable source terms to drive the global solution to an equivalent global fine mesh solution. The method and its implementation have been examined in several test cases and sensitivity studies, and the results consistently demonstrate the validity of the proposed methodology.

\section{ACKNOWLEDGEMENT}

The author gratefully appreciates the initiative and vision in establishing the Chair of Computational Aerothermal Engineering and the stimulating environment of academic freedom and intellectual independence at Oxford, which have made the present work possible.

\section{REFERENCES}

[1] Hughes T.J.R., Mazzei, L., and Jansen K.E., Large Eddy Simulation and the Variational Multiscale Method, Computing and Visualization in Science, Vol.3. No. 1-2, 2000.

[2] Jenny P., Lee, S.H. and Tchelepi, H.A., Multi-scale finite-volume method for elliptic problems in subsurface flow simulation, J. Computational. Physics 187 (2003), pp. 47-67.

[3] Aarnes, J.E., On the Use of a Mixed Multiscale Finite Element Method for Greater Flexibility and Increased Speed or Improved Accuracy in Reservoir Simulation, Multiscale Model. Simulation, 2 (3) (2004), pp. 421-439.

[4] Hou, T.Y, Hu, T.Y, and Hussain Y, Multi-scale Modeling of Incompressible Turbulent Flows, Journal of Computational Physics, 132(1), 2013.

[5] He, L. and Oldfield, M.L.G., Unsteady Conjugate Heat Transfer Modelling, Journal of Turbomachinery, Vol.133. No.3, 2011. 
[6] Errera M, and Turpin G., Temporal Multiscale Strategies for Conjugate Heat Transfer Problems, Journal of Coupled Systems and Multiscale Dynamics, 2013, Vol.1, pp 89-98.

[7] He, L. and Fadl, M., Multi-scale Time Integration for Transient Conjugate Heat Transfer, International Journal of Numerical Methods in Fluids, 2017, Vol.83, Issue 12, pp887904, DOI: 10.1002/fld.4295, Sept, 2016.

[8] He, L., Block-spectral Approach to Film-cooling Modeling, Journal of Turbomachinery, Vol.134 (2), (2012), pp. 021018-1- 021018-8.

[9] He, L. Block-Spectral Mapping for Multi-Scale Solution, J. Comp Phys,, 250 (2013), pp1326, Oct 2013.

[10] He, L. Fourier Spectral Method for Multi-scale Aerothermal Analysis, International Journal of Computational Fluid Dynamics, 2013, Vol.27, No2, pp118-129.

[11] Adamczyk, J. J., Model Equations for Simulating Flows in Multistage-Turbomachinery, ASME Paper 85-GT-226, 1985,

[12] Ning, W., and He, L. Some Modelling Issues on Trailing Edge Vortex Shedding, AIAA Journal, Vol.39, No.5, pp787-793, May, 2001.

[13] Lukovic, B., Orkwis, P. D., Turner, M. G., and Sekar, B., Neural Network Modeling of Deterministic Unsteadiness Source Terms. AIAA Journal, Vol. 44, No. 7, 2006, pp. 16091616. doi: $10.2514 / 1.4222$.

[14] Jameson, A. Time-Dependent Calculations using Multi-Grid, with Applications to Unsteady Flows past Airfoil and Wings, AIAA Paper 91-1596, Jan, 1991.

[15] He, L., New Two-Grid Acceleration Method for Unsteady Navier-Stokes Calculations, AIAA Journal of Propulsion and Power, Vol. 9, No. 2, 1993, pp. 272-280.

[16] He, L., 2000, 3D Navier-Stokes Analysis of Rotor-Stator Interactions in Axial flow Turbines, Journal of Power and Energy, Vol.214, pp13-22.

[17] Liou, M-S, A Sequel to AUSM, Part II: AUSM+-up for all speeds, An Accurate and Robust Flux Splitting Scheme for Shock and Contact Discontinuities, J. of Computational. Physics, Vol. 214 (1), pp.137-170, 2006. 
[18] Cada M. and Torrilhon M, Compact Third-order Limiter Function s for Finite Volume Methods, J. of Computational. Physics, Vol.228(11), pp4118-4145, 2009.

[19] Spalart, P.R. and Allmaras, S.R., A One-equation Turbulence Model for Aerodynamic Flows, AIAA Paper, 92-0439, 1992.

[20] Menter, F. R., Review of the Shear-Stress Transport Turbulence Model Experience from an Industrial Perspective, International J. of Computational. Fluid Dynamics, Vol. 23, Issue 34. 2009.

[21] He, L., Harmonic Solution of Unsteady Flow around Blade with Separation, AIAA Journal, Vol.46, No.6, 2008.

[22] Zhang, M., and He, L., Efficient LES Evaluation of Various Complex Unsteady Flow Configurations in a Design Space, Global Power and Propulsion Society (GPPS) Conference, Paper 0023, Shanghai, 2017.

[23] Denton, J. D., The Calculation of Three-dimensional Viscous Flow through Multistage Turbomachine, ASME Journal of Turbomachinery, Vol.114, No.1, 1992.

[24] Thom, A., The Flow Past Circular Cylinders at Low Speeds. Proceedings of Royal Society of London, Series A, Vol.141, No 845, 1933.

[25] Lad, B. and He, L., Use of an Immersed Mesh for High Resolution Modeling of Film Cooling Flow, ASME Journal of Turbomachinery, Vol.135, No.1, 2012.

[26] Lund, T. S., Wu, X., and Squires, K. D., Generation of Turbulent Inflow Data for SpatiallyDeveloping Boundary Layer Simulations, J. Comp Phys, 140 (1998), pp. 233-258. 